University of Zurich

Department of Economics

Working Paper Series

ISSN 1664-7041 (print)

ISSN 1664-705X (online)

Working Paper No. 385

\title{
Foundations of Pseudomarkets: Walrasian Equilibria for Discrete Resources
}

Antonio Miralles and Marek Pycia

May 2021 


\title{
Foundations of Pseudomarkets: Walrasian Equilibria for Discrete Resources
}

\author{
Antonio Miralles and Marek Pycia* \\ This draft: May 2021. First posted draft: October 2014.
}

\begin{abstract}
We study the assignment of discrete resources in a general model encompassing a wide range of applied environments, such as school choice, course allocation, and refugee resettlement. We allow single-unit and general multi-unit demands and any linear constraints. We prove the Second Welfare Theorem for these environments and a strong version of the First Welfare Theorem. In this way, we establish an equivalence between strong efficiency and decentralization through prices in discrete environments. Showing that all strongly efficient outcomes can be implemented through pseudomarkets, we provide a foundation for using pseudomarkets in market design.
\end{abstract}

\section{Introduction}

Efficiency is the key objective in assignment of discrete resources, or bundles of resources, in environments such as school choice, course assignment, and refugee resettlement. In

*Università degli Studi di Messina - Universitat Autonoma de Barcelona - Barcelona Graduate School of Economics (Miralles), and University of Zurich (Pycia). From 2014 till 2019 this paper was posted and presented under the title "Prices and Efficient Assignments Without Transfers" (first presentation: May 2014, first posted draft: October 2014; substantial revision: February 2017). We would like to thank Salvador Barbera, Roland Benabou, Dirk Bergemann, Simon Board, Sergiu Hart, Andrew McLennan, Andreu MasColell, Jordi Massó, Moritz Meyer-ter-Vehn, Alessandro Pavan, Michael Richter, William Thomson, Utku Unver, Rakesh Vohra, William Zame, Juan Enrique Martínez-Legaz and audiences at UCLA, UAB, SAET 2015, and U Zurich for helpful comments, and Tuomas Kari, Julian Teichgräber, and Simpson Zhang for excellent research assistance. Miralles gratefully acknowledges the hospitality of Ortygia Business School, UCLA, and Carnegie Mellon University, where most of the work has been undertaken, as well as the financial support from the Spanish Governments (SEV-2015-0563, ECO2017-83534P) and the Catalan Government (SGR2017 0711). Pycia gratefully acknowledges the support of UCLA, where he was a faculty member when working on this paper, as well as the support from the William S. Dietrich II Economic Theory Center at Princeton University. 
these environments, the standard stochastic allocation mechanisms that only rely on participants' ordinal rankings over resources or bundles are known to cause efficiency losses. ${ }^{1}$ Since Hylland and Zeckhauser (1979), we know that efficient mechanisms can be constructed by endowing market participants with token money that they can use to buy probability shares in allocated objects, with the allocation determined via Walrasian equilibrium. The resulting mechanisms - known as pseudomarkets - became central to the literature on efficient assignment in discrete environments. ${ }^{2}$

The two main questions the present paper addresses are: how flexible is the pseudomarket approach? in particular, can all efficient assignments be implemented via pseudomarkets? By answering these two questions - and establishing a positive answer to the second one - we provide a foundation for the market design literature's focus on pseudomarkets: in market design contexts, our characterization of efficient assignments allows one to restrict attention to pseudomarkets at least in settings, such as large markets, where pseudomarket price mechanisms are incentive compatible. ${ }^{3}$ This positive answer is tantamount to proving for general discrete allocation environments an analogue of the Second Welfare Theorem of the classic Walrasian theory: that every Pareto efficient assignment can be decentralized through the use of prices. In classic Walrasian markets, at least one good is divisible, and agents always strictly prefer having more of this good; in reduced-form models of markets the role of such a good is played by numeraire or money. The presence of such numeraire good implies that each agent is locally non-satiated, that is, for any assignment there is a nearby assignment that the agent strictly prefers. In contrast, our agents may be satiated if they receive their most preferred bundles.

We establish a tight link between efficiency and pseudomarkets despite the failure of

\footnotetext{
${ }^{1}$ Such losses are particularly pronounced when market participants have multi-unit demands as established by Budish and Cantillon (2012) and Budish (2011) in the course allocation context. The losses are also present in single-unit demand environments such as school choice: Bogomolnaia and Moulin (2001), Abdulkadiroglu, Che, and Yasuda (2011), Featherstone and Niederle (2016), Miralles (2008), and Pycia (2014) provide theoretical analyses of such losses, and Abdulkadiroglu, Agarwal, and Pathak (2017) provide their empirical evaluation. While deterministic mechanisms fare better - unlike stochastic mechanisms they can be Pareto efficient - in many environments stochasticity plays an important role, for instance because of fairness considerations, cf. Abdulkadiroglu and Sonmez (2003) and Abdulkadiroglu, Pathak, and Roth (2009). Refugee resettlement entails the allocation of discrete resources to the refugee families, and ordinal allocation mechanisms inherit the efficiency losses first established in school choice and course allocation studies; for a discussion of these mechanisms in refugee context, see e.g. Andersson and Ehlers (2020), Delacretaz, Kominers, and Teytelboym (2020), and Pycia (2019). Even allocating legal tender money is a special case of our setting as long as the money comes in a finite number of discrete coins and banknotes; however the cited analyses of efficiency losses of ordinal mechanisms relied on the absence of legal tender.

${ }^{2}$ We provide a review of this rich literature below.

${ }^{3}$ See He et al. (2018) for asymptotic strategy-proofness of pseudomarkets, Azevedo and Budish (2019) for their strategy-proofness in the large, and Pycia (2014) for Nash equilibria. While these papers assume that participants' budgets are fixed, in an ongoing work we show that this assumption may be relaxed.
} 
local non-satiation. The feature of the environment that enables this unexpected link is the discreteness of resources being allocated; otherwise our allocation model is general. There is a finite set of agents and objects. Agents are assigned bundles of objects and we impose no assumptions on agents' utilities from the bundles. Lotteries over bundles are evaluated in line with the expected utility theory. As we allow for arbitrary multi-unit demands, our model accommodates as special cases all types of substitutes, complements, externalities among objects in the same bundle, as well as the canonical single-unit demand model of Hylland and Zeckhauser (1979). Extending Hylland and Zeckhauser's pseudomarkets to our general setting, we study Walrasian equilibria in which each agent is endowed with token money; the amount of token money held after the assignment has no impact on agents' utilities. $^{4}$

Our main result takes a particularly simple form in the single-unit demand settings such as school choice: every Pareto efficient assignment may be supported in a Walrasian equilibrium with properly chosen budgets, and hence decentralized via prices. The link between efficiency and prices remains valid in the general multi-unit-demand random assignment model in which agents receive lotteries over bundles of indivisible goods. In the general multi-unit-demand case the statement of this link is however more subtle because - as we show in an example - there are environments in which some assignments are Pareto efficient, in the sense of being undominated by any feasible random assignment, and at the same time these assignments cannot be supported in any Walrasian equilibrium. ${ }^{5}$ We thus prove the Second Welfare Theorem for allocations that are strongly Pareto efficient in the following sense: they are undominated by random allocations that are feasible at least in expectation. ${ }^{6}$ Importantly, we prove that strong efficiency is not only sufficient but also necessary for the Second Welfare Theorem, that is we also prove the analogue of the First Welfare Theorem for strong efficiency: every Walrasian equilibrium is efficient in the strong sense. ${ }^{7}$

\footnotetext{
${ }^{4}$ For earlier extensions of Hylland and Zeckhauser's idea to multi-unit demand settings, see Budish (2011) and Budish et al. (2013). In addition to establishing the Second Welfare Theorem in their environments, we relax the modeling restrictions their analyses rely on.

${ }^{5}$ The subtlety is caused by the failure of the Birkhoff-von Neumann property: in general random allocations whose expectations are feasible may fail to be implementable as a lottery over feasible deterministic assignments. Cf. Budish et al. (2013) and Nguyen, Peivandi and Vohra (2016) for a discussion of failures of the Birkhoff-von Neumann property. In all environments in which Birkhoff-von Neumann property obtains - in particular in environments studied by Budish et al. (2013) - our results show that every Pareto efficient assignment may be supported in a Walrasian equilibrium.

${ }^{6}$ Our Second Welfare Theorem implies as a corollary that whenever feasibility in expectation is the relevant feasibility concept, then the Second Welfare Theorem holds true for standard Pareto efficiency. This is of relevance in large markets as Nguyen, Peivandi and Vohra (2016) extended the Birkhoff-von Neumann Theorem to multi-unit assignment in large markets showing that the set of feasible-in-expectation random assignments is asymptotically equivalent to the set of implementable random assignments. Following on our analysis, Miralles and Pycia (2017) identify a sufficient condition for the Second Welfare Theorem to obtain in multi-unit-demand environments with divisible goods, possibly nonlinear preferences, and agents demanding goods up to a capacity quota (and hence possibly satiated).

${ }^{7}$ For the school choice setting, the First Welfare Theorem was established by Hylland and Zeckhauser
} 
The conjunction of our First and Second Welfare Theorems establishes the equivalence of pseudomarkets and strong efficiency, thus answering the first of our two leading questions, how flexible pseudomarkets are.

Whether the Second Welfare Theorem obtains in discrete settings was a puzzle, except for deterministic assignments in single-unit demand settings, for which Abdulkadiroglu and Sonmez (1998) established a version of the Second Welfare Theorem. We find it quite surprising that the insight of the Second Welfare Theorem holds true in discrete environments because the problems the received approaches to the Second Welfare Theorem run into in settings with non-divisibilities and locally satiated agents are well-known (Mas-Collel, Winston, and Green, 1995). The failure of local non-satiation implies that the Separating Hyperplane Theorem commonly used to prove the Second Welfare Theorem guarantees only the existence of a separating hyperplane that may have non-empty intersections with the set of Pareto-dominant aggregate assignments. ${ }^{8}$ Facing the resulting prices, some agents might afford to buy bundles they strictly prefer over their assignment; this situation is called a quasi-equilibrium.

To surmount the problems that satiation causes for the standard proof approach, we develop a novel approach to constructing the separating hyperplane that leverages the polytope properties of discrete environments. As a key part of our proof, we establish a Full Separation Lemma for Polytopes that might be useful beyond the confines of our Walrasian analysis. ${ }^{9}$ The lemma establishes the existence of a separating hyperplane that is disjoint with the set of Pareto-dominant aggregate assignments. Facing the resulting prices, no agent can afford a bundle they would prefer over their assignment, and the prices support the assignment as an equilibrium. To the best of our knowledge, ours is the first paper to leverage the properties of the polytopes to analyze Walrasian equilibria and prove the Second Welfare Theorem. ${ }^{10}$

Prior work on implementing efficient outcomes via pseudomarkets relied on additional strong requirements. In continuum economies, Thomson and Zhou (1993) related efficient, symmetric, and consistent mechanisms to Hylland and Zeckhauser's pseudomarket mecha-

(1979). This result was further refined and extended by Mas-Collel (1992) and Budish, Che, Kojima, and Milgrom (2013). For instance, all equilibria are efficient if agents strictly rank any two objects. Note that the validity of the First Welfare Theorem in some of the settings we study does not imply the validity of the Second Welfare Theorem for these settings; indeed, there are environments in which the First Welfare Theorem holds true, and the Second Welfare Theorem fails, cf. Mas-Collel, Whinston, and Green (1995).

${ }^{8}$ While the full separation obtains if one of the separated sets is open, this assumption fails in our setting. Section 3 provides an example illustrating the failure of openness and a more detailed discussion of why the standard techniques do not work.

${ }^{9}$ We also prove a complementary Polytope Lemma that shows that the set of Pareto dominant outcomes is a polytope, provided the resources being allocated are discrete.

${ }^{10}$ For earlier uses of polytope ideas to study other questions in economics, see, e.g., McLennan (2002), Budish et al. (2013), Pycia and Unver (2015); none of these papers analyzes Walrasian equilibria. 
nism with equal budgets, and Ashlagi and Shi (2016) showed that any efficient, symmetric, and strategy-proof random assignment can be expressed as the result of the equalbudget pseudomarket mechanism. ${ }^{11}$ In contrast, we do not rely on symmetry, consistency, or strategy-proofness, and we prove our results for all finite economies.

Our paper also contributes to the literatures on constraints in market design - e.g., Budish, Che, Kojima, and Milgrom (2013) and He, Miralles, Pycia, and Yan (2018) — and on multi-unit assignment - e.g., Sonmez and Unver (2010), Budish (2011), and Budish and Cantillon (2012) - that extended the idea of using token money to allocate objects beyond the canonical Hylland and Zekchauser setting. ${ }^{12}$ Our Second Welfare Theorem is complementary to these papers and provides a microfoundation for their focus on pseudomarkets; none of these earlier papers provided such a microfoundation. We also improve upon the First Welfare Theorems established in these papers by showing that pseudomarket equilibria are not only Pareto efficient but also strongly efficient, and our general multi-unit demand setting goes beyond the settings studied in these papers: our analysis allows arbitrary utility profiles over bundles of objects and arbitrary linear constraints. In particular, our Second Welfare Theorem does not hinge on the standard assumption that goods are substitutes, and it allows any mixture of substitutes and complementarities. ${ }^{13}$

Our paper provided a microfoundation for the focus on pseudomarkets in the analysis of efficient mechanisms also for the many papers that followed on our work. Papers that crucially rely on our Second Welfare Theorem include Miralles and Pycia (2015), who address the question which assignments are efficient and envy-free and show that the answer is qualitatively different in large finite markets than in a continuum economy limit, as well as Miralles (2017) and Schlegel and Mamageishvili (2019), who study He et al's (2018) pseudomarkets with weak priorities. Other papers that followed on our work and whose focus on pseudomarkets is microfounded by our Second Welfare Theorem include Babaioff, Nisan, Talgam-Cohen (2018), McLennan (2018), Echenique, Miralles and Zhang (2019a, 2019b), and Gul, Pesendorfer and Zhang (2019); the focus of these papers is on equilibrium existence,

\footnotetext{
${ }^{11}$ Miralles and Pycia (2015) showed that the latter result hinges on the presence of the continuum of agents and Hafalir and Miralles' (2015) study more demanding utilitarian welfare. Cf. also Makowski, Ostroy, and Segal (1999) for classical exchange economies. Subsequent to our work, Bogomolnaia et al. (2017, 2019) show that the utility profile of the equal-budget pseudomarket mechanisms maximize the Nash product of utilities; in particular the resulting profile is fully determined by the set of feasible utility profiles.

${ }^{12}$ For analysis of market design constraints beyond the token money mechanisms, see also e.g. Pycia and Unver (2015), and Kojima and Kameda (2015). Beyond allocation, the token money ideas were used e.g. in Manjunath's (2014) analysis of two-sided matching.

${ }^{13}$ In this sense we are also contributing to the literature extending the economic analysis of matching and allocation models beyond the standard substitutes assumption; cf. Sun and Yang (2006), Ostrovsky (2008), Pycia (2012), Baldwin and Klemperer (2019) for earlier analyses going beyond the substitute assumption. At the current still early stage of this literature and the literature on constraints, they focus primarily on existence results most closely related to our secondary result, the First Welfare Theorem.
} 
particularly in the context of fairness requirements or in the presence of constraints. ${ }^{14}$

Finally, we contribute to the literature on the Second Welfare Theorem, also known as the Second Fundamental Theorem of Welfare Economics. The theorem was conjectured by Pareto (1909), and subsequently refined and developed by many authors. Arrow (1951) proved the theorem assuming strict concavity of preferences and Debreu (1951) proved it assuming weak concavity and local non-satiation. Anderson (1988) allowed nonconvex preferences but maintained the assumption of local non-satiation. Florig and Rivera (2010) established an almost-everywhere Second Welfare Theorem for large markets with a continuum of agents; in contrast, our analysis is valid in finite markets. Richter and Rubinstein (2015) proposed a general convex geometry approach to welfare economics based on the concept of "primitive equilibrium," where a strict linear ordering arranges alternatives in order to create "budget" sets. They proved a Second Welfare Theorem for the primitive equilibrium concept; when preferences are strictly monotone, their primitive equilibrium concept corresponds to the standard equilibrium concept; however, when specialized to our setting, this equilibrium concept becomes equivalent to the quasi-equilibrium discussed above. ${ }^{15}$

\section{Base Model}

We study a finite economy with agents $i, j \in I=\{1, \ldots,|I|\}$ and indivisible objects $x, y \in$ $X=\{1, \ldots,|X|\}$. Each object $x$ is represented by a number of identical copies $|x| \in \mathbb{N}$. By $S=(|x|)_{x \in X}$ we denote the total supply of object copies in the economy. If agents have outside options, we treat them as objects in $X$; in particular, this implies that $\sum_{x \in X}|x| \geq|I|$. We assume initially that agents demand at most one copy of an object; we fully relax this assumption in Section 4. A canonical interpretation of the single-unit demand model is school choice, in which each student demands entry to at most one school.

We allow random assignments and denote by $q_{i}^{x} \in[0,1]$ the probability that agent $i$ obtains a copy of object $x$. Agent $i$ 's random assignment $q_{i}=\left(q_{i}^{1}, \ldots, q_{i}^{|X|}\right)$ is a probability distribution. The economy-wide assignment $Q=\left(q_{i}^{x}\right)_{i \in I, x \in X}$ is feasible if the aggregate assignment (which we will denote as $A(Q)$ ) is weakly lower that the supply vector: $A(Q) \equiv$

\footnotetext{
${ }^{14}$ Of interest is also Reny (2017), who extends the deterministic analysis of Budish (2011) beyond discrete outcomes spaces, as well as Baldwin et al. (2020), who study the First Welfare Theorem, and Vazirani and Yannakakis (2020), who study the complexity of pseudomarket mechanisms.

${ }^{15}$ In Section 3 we provide an example of a quasi-equilibrium which is not an equilibrium; this quasiequilibrium is a primitive equilibrium in the sense of Richter and Rubinstein. To the best of our knowledge the above discussion covers all extensions of the Second Welfare Theorem beyond Arrow and Debreu. Of course, the literature on Walrasian equilibria beyond this setting is richer, and - in addition to the papers cited above -includes, for instance, Bergstrom (1976), Manelli (1991), and Hara (2005) who focused on equilibrium existence and core convergence rather than on the Second Welfare Theorem.
} 
$\sum_{i \in I} q_{i} \leq S$. Let $\mathcal{A}$ denote the set of economy-wide random assignments, and $\mathcal{F} \subset \mathcal{A}$ denote the set of feasible random assignments. We call an assignment pure, or deterministic, if each of its elements $q_{i}^{x}$ is either 0 or 1 . By the Birkhoff-von Neumann theorem, a feasible random assignment can be expressed as a lottery over feasible pure assignments.

Agents are expected utility maximizers, and agent $i$ 's utility from random assignment $q_{i}$ equals the scalar product $u_{i}\left(q_{i}\right)=v_{i} \cdot q_{i}$ where $v_{i}=\left(v_{i}^{x}\right)_{x \in X} \in[0, \infty)^{|X|}$ is the vector of agent $i$ 's von Neumann-Morgenstein valuations for objects $x \in X$.

We study the connection between two concepts: efficiency and equilibrium. A feasible random assignment $Q^{*} \in \mathcal{F}$ is ex-ante Pareto efficient-or, simply, efficient-if no other feasible random assignment $Q \in \mathcal{F}$ is weakly preferred by all agents and strictly preferred by some agents.

A random assignment $Q^{*} \in \mathcal{F}$ and a price vector $p^{*} \in \mathbb{R}^{X}$ constitute an equilibrium (or Walrasian equilibrium) for a budget vector $w^{*} \in \mathbb{R}_{+}^{|I|}$ if $Q^{*}=\left(q_{i}^{*}\right)_{i \in I}$ is feasible in the sense $p^{*} \cdot q_{i}^{*} \leq w_{i}^{*}$ for all $i \in I$, and $u_{i}\left(q_{i}\right)>u_{i}\left(q_{i}^{*}\right) \Longrightarrow p^{*} \cdot q_{i}>w_{i}^{*}$ for all $\left(q_{i}\right)_{i \in I} \in \mathcal{A}$.

\section{The Second Welfare Theorem for Single-Unit De- mand}

We now develop the Second Welfare Theorem for agents with single-unit demand. The result is directly applicable to school choice. Furthermore, the analysis serves as an example illustrating the approach that in the next section we use to derive a general Second Welfare Theorem for assignment with multi-unit demand.

Theorem 1. (The Second Welfare Theorem for Single-Unit Demand) If $Q^{*} \in \mathcal{F}$ is Pareto-efficient, then there is a vector of budgets $w^{*} \in \mathbb{R}_{+}^{|I|}$ and a vector of prices $p^{*} \in \mathbb{R}_{+}^{|X|}$ such that $Q^{*}$ and $p^{*}$ constitute an equilibrium with budgets $w^{*}$.

Before laying out the proof, let us compare our problem to the standard Second Welfare Theorem for agents whose preferences are convex and strictly monotonic. The well-known argument in the standard setting relies on the celebrated separating hyperplane theorem: for any two disjoint convex sets $Y, Z \subseteq \mathbb{R}^{n}$ there exists a price vector $p \in \mathbb{R}^{n}$ and budget $w \in \mathbb{R}$ such that $p \cdot z \geq w \geq p \cdot y$ for each $z \in Z$ and $y \in Y$, thus achieving a partial separation of $Y$ and $Z$; the separation is full if one of the inequalities can be assumed to be strict. ${ }^{16}$ In the standard proof, $Y$ is the set of aggregate feasible assignments and $Z$ is the

\footnotetext{
${ }^{16}$ See e.g. Boyd and Vandenberghe (2004). An alternative proof of the standard Second Welfare Theorem was offered by Maskin and Roberts (2008): their "revealed preference" approach is inapplicable in our
} 
set of (infeasible) aggregate assignments that Pareto dominate a fixed efficient assignment $Q^{*}=\left(q_{i}^{*}\right)_{i \in I}$ we want to implement. ${ }^{17}$ If now some agent $i \in I$ strictly prefers some $q_{i}$ to $q_{i}^{*}$, then $Q=\left(q_{i}, q_{-i}^{*}\right)$ Pareto dominates $Q^{*}$ and by the partial separation inequality, $p \cdot\left(q_{i}+\sum_{j \in I \backslash\{i\}} q_{j}^{*}\right) \geq w \geq p \cdot \sum_{j \in I} q_{j}^{*}$, where the second inequality can be shown to be an equality. Setting $w_{i}=p \cdot q_{i}^{*}$ we conclude that

$$
u_{i}\left(q_{i}\right)>u_{i}\left(q_{i}^{*}\right) \Longrightarrow p^{*} \cdot q_{i}>w_{i}^{*}
$$

thus prices $p$ and budgets $w_{i}$ give us a so-called quasi-equilibrium .

The key step of the standard proof is then to show that the above quasi-equilibrium is in fact an equilibrium, that is

$$
u_{i}\left(q_{i}\right)>u_{i}\left(q_{i}^{*}\right) \Longrightarrow p^{*} \cdot q_{i}>w_{i}^{*}
$$

for all $i \in I$ and for all $\left(q_{i}\right)_{i \in I} \in \mathcal{A}$. This last step is by contradiction: we take an assignment $Q=\left(q_{i}\right)_{i \in I}$ that Pareto dominates $Q^{*}$ while there is an agent $i$ for whom $q_{i}$ costs the same as $q_{i}^{*}$; in the neighborhood of $Q$ we then find an assignment that still Pareto dominates $Q^{*}$ while being cheaper than it. This is a contradiction as in quasi-equilibrium no cheaper assignment can Pareto dominate $Q^{*}$.

It is this key step of the standard proof that fails in our setting. The standard separating hyperplane theorem partially separates the Pareto dominating aggregate assignments from the feasible ones. In the standard argument this is sufficient because the set of Pareto dominating aggregate assignments is open; in contrast, in the setting we study, this set of aggregate assignments does not need to be open. In effect, while full separation follows from the partial one (and hence every quasi-equilirbium is an equilibrium) in the standard setting, in the discrete setting with locally satiated preferences that we study, the full separation does not follow from the partial one and not every quasi-equilibrium is an equilibrium. The standard argument breaks at the claim that there is a cheaper but still Pareto-dominant assignment; this step relies on the prices of goods being strictly positive, which obtains in the standard setting as otherwise agents would demand an infinite amount of zero-price goods. In contrast, zero prices are the staple of our setting as recognized already by Hylland and Zeckhauser (1979). In particular, in a quasi-equilibrium an agent may be assigned a

setting because it relies on endowing agents with initial shares in objects as opposed to token budgets. Pseudomarkets do not allow share endowments for reasons explicated in Hylland and Zeckhauser (1979). Furthermore, Maskin and Roberts (2008) rely on local non-satiation and the resulting property that any two bundles an agent is indifferent between have the same price; both local non-satiation and the same-price property fail in our setting. The failure of the latter is illustrated in footnote 26 .

${ }^{17}$ Note that these sets are convex and they are disjoint. 


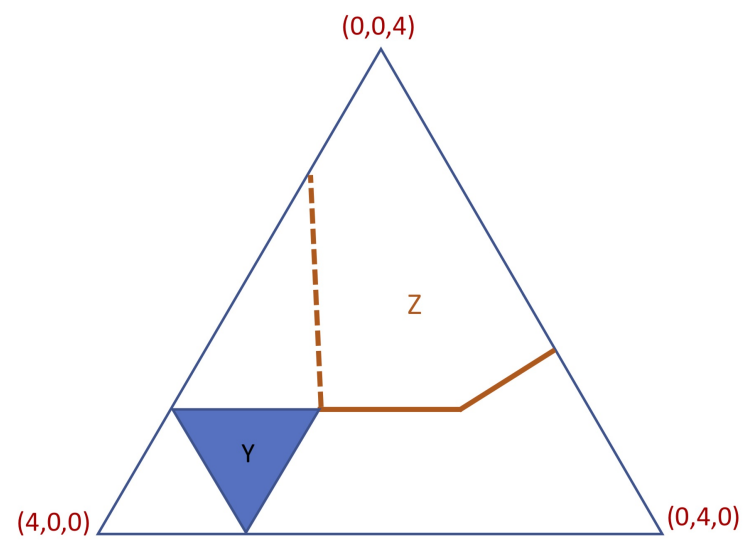

Figure 1: The simplex of "full-consumption" aggregate assignments. Aggregate assignment $A\left(Q^{*}\right)$ is on the intersection of the boundaries of sets $Y$ and $Z$.

zero-price object while he strictly prefers another zero-price object.

As an illustration of these problems, consider the following example.

Example 1. Consider an economy with four agents and three objects. Two of the agents have von Neumann-Morgenstern utility vector $v=\left(\frac{1}{2}, 0,1\right)$, and the remaining two agents have the utility vector $v^{\prime}=\left(0,1, \frac{1}{2}\right)$. Suppose that there are three copies of object 1 , one copy of object 2, and one copy of object 3. The following allocation $Q^{*}$ is then Pareto-efficient: $v$-agents obtain $q^{*}=\left(\frac{1}{2}, 0, \frac{1}{2}\right)$ and $v^{\prime}$-agents obtain $q^{* \prime}=\left(\frac{1}{2}, \frac{1}{2}, 0\right)$.

The resulting aggregate assignment $A\left(Q^{*}\right)$ is $(2,1,1)$. Figure 1 places this point in the barycentric simplex of aggregate assignments in which exactly four units are assigned, that is such that for each agent the sum of probabilities of the three goods is 1 (the full-consumption simplex). Set $Y$ represents feasible aggregate assignments in the simplex; it is the triangle spanned by $(2,1,1),(3,0,1)$ and $(3,1,0)$. Set $Z$ represents all aggregate assignments $A(Q)$ in the simplex such that there exists an assignment $Q$ in which all agents are weakly better off than under $Q^{*}$ and at least one agent is strictly better off, and such that $A(Q)$ is the aggregate assignment of $Q$ (these assignments are, of course, not feasible). Set $Z$ has five corners:

- $(2,1,1)$, the aggregate assignment corresponding to $Q^{*}$,

- $(1,2,1)$, the aggregate assignment when $v$-agents obtain $q^{*}$ and $v^{\prime}$-agents obtain $(0,1,0)$,

- $\left(0,2 \frac{1}{2}, 1 \frac{1}{2}\right)$, the aggregate assignment when $v$-agents obtain $\left(0, \frac{1}{4}, \frac{3}{4}\right)$ and $v^{\prime}$-agents obtain $(0,1,0)$,

- $(0,0,4)$, the aggregate assignment when each agent obtains good 3 
- $(1,0,3)$, the aggregate assignment when $v$-agents obtain $q^{*}$ and $v^{\prime}$-agents obtain $(0,0,1)$.

Only the middle three corners belong to $Z$, and one of the borders of $Z$, the dashed line, is disjoint with $Z$. In particular, the set $Z$ is neither open nor closed.

Restricting attention to the assignments in the simplex, there is a horizontal hyperplane separating $Y$ and $Z$. This hyperplane corresponds to prices $p^{3}>p^{2}=p^{1}=0$. When $v$-agents have budget $\frac{1}{2} p^{3}$ and $v^{\prime}$-agents have budget zero, these prices support $Q^{*}$ as a quasi-equilibrium but not as an equilibrium. Indeed, $v^{\prime}$-agents would rather buy a sure copy of object 2 than the lottery $q^{* \prime}$, and both these outcomes have the price of zero. ${ }^{18}$

We develop a new proof approach to establish the second welfare theorem and to address the difficulties discussed above and illustrated in Example 1. To understand our approach, observe that in Example 1, there are non-horizontal hyperplanes that fully separate $Y$ and $Z$ (in the full-consumption simplex). We show that this is always the case. A key step in the proof is the following new Full Separation Lemma that establishes that full separation is possible under conditions that - as we will shortly see - are always satisfied in the discrete assignment problems. The full separation relies on the assumption that some of the relevant sets are polytopes, where a polytope is the intersection of a finite number of half spaces. ${ }^{19}$

Lemma 1. (Full Separation Lemma) Let $Y \subset \mathbb{R}^{n}$ be a closed and convex polytope. Let $Z \subset \mathbb{R}^{n}$ be convex, non-empty, and such that its closure $\bar{Z} \subset \mathbb{R}^{n}$ is a closed and convex polytope. Suppose that $Z \cap Y=\emptyset$ and that for all $y \in Y \cap \bar{Z}, \delta \in \mathbb{R}^{n}$, and $\varepsilon>0$ if $y+\delta \in Z$, then $y-\varepsilon \delta \notin \bar{Z}$. Then, there exists a price vector $p \in \mathbb{R}_{+}^{n}$ and a budget $w \in \mathbb{R}$ such that for

\footnotetext{
${ }^{18}$ As perceptively observed by a referee, this example has several features that might lead one to wonder whether the problems illustrated by the example can be avoided if we restrict attention to strictly positive valuations or require non-zero budgets. Such simple solutions would not address the problems illustrated by Example 1. For instance, we can add any constant to the valuations and multiple it by any scalar and in such modified example the problematic partially separating hyperplane would still be present even though all valuations are then strictly positive. We could endow $v^{\prime}$-type agents with any positive budget without otherwise changing the example, and the problematic hyperplane would still be there. We could also modify the example so that all agents have strictly positive budgets and fully spent them: e.g. we could enrich the example by adding a fourth good, which only has one unit available, and that types $v^{\prime}$ like more than other goods (and types $v$ do not want to buy); in such a modification of the example, the price of the fourth good would be strictly positive and equal to twice $v^{\prime}$ types' individual budgets and the problematic hyperplane would still be present.

${ }^{19}$ The terminology varies in the literature, with some authors referring to this concept as polyhedra and reserving the term polytope for compact polyhedra. We use the polytope in the above broader sense; in particular, our lemma does not rely on compactness. In the proof of our lemma we rely on an elegant Polytope Separation Lemma that McLennan (2002) developed in an ordinal context unrelated to the problems studied in our paper, and that was never previously used to analyze Walrasian equilibria. McLennan's lemma cannot be substituted for our Full Separation Lemma in the simple proof of our Second Welfare Theorem presented below because his lemma establishes only partial separation between polytopes, while our proof relies on full separation established by our lemma. (The December 2014 draft of our paper sketched an alternative direct proof of our Full Separation Lemma, and we would like to thank Andrew McLennan for directing us to his lemma as a basis for the current simplified version of our proof).
} 
any $z \in Z$ and $y \in Y$ we have $p \cdot z>w \geq p \cdot y$ and such that for any $\bar{z} \in \bar{Z}$ and $y \in Y$ we have $p \cdot \bar{z} \geq w \geq p \cdot y$.

We provide the proof of the lemma in Appendix A.

We can easily visualize the statement of the lemma in the context of Example 1. Both the set $Y$ of feasible aggregate assignments and the set $Z$ of (infeasible) aggregate assignments that Pareto dominate $Q^{*}$ are polytopes. Our separation lemma states that if every line through $Q^{*}$ and a point in $Z$ has points that belong to the closure of $Z$ only on one side of $Q^{*}$, then there exists a fully separating hyperplane. The line assumption is satisfied in our example.

The rest of the proof of the second welfare theorem revolves around showing that indeed the assumption of the lemma is satisfied: no line through $Q^{*}$ can intersect the closure of $Z$ on both sides of $Q^{*}$ (see the highlighted claim in the proof below).

Proof of the Second Welfare Theorem. For any random assignment $Q \in \mathcal{A}$, we define the aggregate assignment $A(Q)$ associated with $Q$ to be $\sum_{i \in I} q_{i}$, and we write $Q \succ Q^{*}$ when $u_{i}\left(q_{i}\right) \geq u_{i}\left(q_{i}^{*}\right)$ for every $i \in I$ with at least one strict inequality.

Let $Z=\left\{A(Q): Q \succ Q^{*}, Q \in \mathcal{A}\right\}$, and notice that the above assumption implies that $Z$ is non-empty. Furthermore, $Z$ is convex. Let $\bar{Z}=\mathrm{Cl}(Z)$ be the topological closure of $Z$, and notice that $\bar{Z}$ is a non-empty convex polytope. Let $Y=\{A(Q): Q \in \mathcal{F}\}$ be the set of aggregate feasible random assignments. This set is a closed and convex polytope, and the efficiency of $Q^{*}$ implies that $Z \cap Y=\varnothing$.

To use the full separation lemma, we need the following

Claim. For any $y \in Y \cap \bar{Z}, \delta \in \mathbb{R}^{|X|}$ and $\varepsilon>0$, if $y+\delta \in Z$ then $y-\varepsilon \delta \notin \bar{Z}$.

Proof of the claim: If $y+\delta \in Z$ then there is a $Q \succ Q^{*}$ such that $A(Q)=y+\delta$. By way of contradiction, assume $y-\varepsilon \delta \in \bar{Z}=\mathrm{Cl}(Z)$. Thus, there is a $\tilde{Q}=\left(\tilde{q}_{i}\right)_{i \in I}$ such that $u_{i}\left(\tilde{q}_{i}\right) \geq u_{i}\left(q_{i}^{*}\right)$ for every $i \in I$ and $A(\tilde{Q})=y-\varepsilon \delta$. Then, the random assignment $\bar{Q}=\frac{\varepsilon}{1+\varepsilon} Q+\frac{1}{1+\varepsilon} \tilde{Q}$ is feasible, and the choice of $Q$ and $\tilde{Q}$ and the linearity of utility $u_{i}(\cdot)$ in probabilities imply that $\bar{Q} \succ Q^{*}$. But this contradicts the fact that $Q^{*}$ is efficient, proving the claim.

This claim and the full separation lemma imply that there exists a price vector $p \in \mathbb{R}_{+}^{|X|}$ and a budget $w \in \mathbb{R}$ such that $p \cdot z>w \geq p \cdot y$, for any $z \in Z$ and $y \in Y$. Since $Q^{*}$ is feasible $\sum_{i \in I} q_{i}^{*} \in Y$ and thus $p \cdot \sum_{i \in I} q_{i}^{*} \leq w$. Furthermore, $p \cdot \sum_{i \in I} q_{i}^{*} \geq w$ because $Q^{*} \in \mathrm{Cl}(Z)$. We conclude $p \cdot \sum_{i \in I} q_{i}^{*}=w$. Now, if we take some $q_{i}$ that some agent $i \in I$ strictly prefers to $q_{i}^{*}$, then $q_{i}+\sum_{j \in I \backslash\{i\}} q_{j}^{*} \in Z$, and we have $p \cdot\left(q_{i}+\sum_{j \in I \backslash\{i\}} q_{j}^{*}\right)>w=p \cdot\left(q_{i}^{*}+\sum_{j \in I \backslash\{i\}} q_{j}^{*}\right)$. Consequently we have $p \cdot q_{i}>p \cdot q_{i}^{*}$, proving that $p$ and $Q^{*}$ constitute an equilibrium for budgets $w_{i}^{*}=p \cdot q_{i}^{*}$. QED 


\section{Multi-Unit Demand: Second and First Welfare The- orems}

We now analyze the validity of our Second Welfare Theorem result in assignment economies in which participants demand multiple units of goods. As in the base model, we have a set of agents $I$ and a set of objects $X$. Each object $x \in X$ has a finite number of units (or copies) $|x| \in\{1,2, \ldots\}$ and $S=(|x|)_{x \in X}$ is the supply vector. ${ }^{20}$ We relax the restriction that each agent demands at most one unit of goods in total, and allow each agent to demand at most $k \in\{1,2, \ldots\}$ units of various goods in total. We impose no restrictions on the positive integer $k$; in particular, it can be larger than the total supply of objects.

Let $B_{i} \subseteq\{0,1, \ldots, k\}^{|X|}$ be the finite set of admissible individual bundles for agent $i$, and let $b_{i 1}, \ldots, b_{i\left|B_{i}\right|}$ denote the elements of $B_{i}$. The set $B_{i}$ can accommodate any restrictions such as, for instance, that the agent consumes at most quantity 1 of each object. Course allocation is an example of a multi-unit demand setting that satisfies this particular restriction: in course allocation, $B_{i}=\left\{b \in\{0,1\}^{|X|}: \sum_{x \in X} b^{x} \leq k\right\}$ for each agent $i$.

An individual random assignment $q_{i} \in \Delta\left(B_{i}\right)$ of agent $i \in I$ is a probability distribution over $B_{i}$. The agent's expected utility is the scalar product $q_{i} \cdot v_{i}$ where $v_{i} \in \mathbb{R}^{\left|B_{i}\right|}$ is the vector of valuations for each bundle in $B_{i}$. For the sake of linear algebra calculations, we represent the set of bundles $B_{i}$ by the matrix $\beta_{i}=\left(\beta_{i b}^{x}\right)_{x \in X, b \in B_{i}}$ in which $\beta_{i b}^{x}$ is the quantity of object $x$ in bundle $b$.

A deterministic assignment of bundles $D=\left(b_{i}\right)_{i \in I} \in \times_{i \in I} B_{i}$ is feasible if $\sum_{i \in I} b_{i} \leq S$, coordinatewise. We denote by $\mathcal{D}$ the (finite) set of all feasible deterministic assignments of bundles and by $b_{i}(D)$ the bundle that agent $i$ obtains under the $D \in \mathcal{D}$. We assume throughout that set $\mathcal{D}$ is non-empty. Denoting $B=\cup_{i} B_{i}$, a random assignment of bundles $Q=\left(q_{i}^{b}\right)_{i \in I, b \in B} \in[0,1]^{I \times B}$ is feasible in expectation if each $q_{i}$ has support on $B_{i}$ and the expected aggregate assignment does not exceed supply for any good, $\sum_{i \in I, b \in B} q_{i}^{b} b \leq$ $S$. A random assignment $Q=\left(q_{i}^{b}\right)_{i \in I, b \in B}$ is feasible (or implementable) if there are nonnegative weights $\left(\lambda_{D}\right)_{D \in \mathcal{D}} \geq 0$ summing up to 1 and such that, for every $i \in I$ and $b \in B, \sum_{b_{i}(D)=b} \lambda_{D}=q_{i}^{b}$. By $\mathcal{F}$ we denote the set of all feasible random assignments. Of course, every feasible assignment is feasible in expectation.

A random assignment of bundles $Q$ ex-ante Pareto-dominates a random assignment of bundles $Q^{*}$ if $q_{i} \cdot v_{i} \geq q_{i}^{*} \cdot v_{i}$ for all $i \in I$, with at least one strict inequality. A feasible random assignment of bundles $Q^{*}=\left\{q_{i}^{*}\right\}_{i \in I}$ is (ex-ante Pareto) efficient if it is not ex-ante Pareto-dominated by any feasible random assignment of bundles. A random assignment of bundles $Q^{*}$ is an equilibrium assignment with prices $p^{*} \in \mathbb{R}_{+}^{|X|}$ and budgets $\left(w_{i}^{*}\right)_{i \in I} \in \mathbb{R}_{+}^{|I|}$

\footnotetext{
${ }^{20}$ Without affecting the results, we can allow $|x|=0$ for all but one object.
} 
if, for every agent $i \in I, p^{*} \cdot \beta_{i} q_{i}^{*} \leq w_{i}$ and if $q_{i} \cdot v_{i}>q_{i}^{*} \cdot v_{i}$ for some random assignment $Q$ then $p^{*} \cdot \beta_{i} q_{i}>w_{i}$.

Our single-unit demand Second Welfare Theorem immediately implies the multi-unit demand Second Welfare Theorem if we allowed separate prices for all bundles. Indeed, then we can think of agents as having a single-unit demand: each of them demands at most one bundle.

The analysis becomes more subtle if we require - as in the definition of the competitive equilibrium above - that the price of a bundle is the sum of prices of the component goods of the bundle. ${ }^{21}$ We can then still apply our Full Separation Lemma and replicate the singleunit demand analysis provided every random assignment that is feasible in expectation is feasible. This property - established in the single-unit case in the Birkhoff-von Neumann Theorem - ensures that if we moved from an initial (feasible) aggregate assignment in some direction to a (non-feasible) Pareto-dominating aggregate assignment, then when moving in the opposite direction the assignments are not weakly Pareto dominant as otherwise a proper linear combination of both assignments would be feasible by the Birkhoff-von Neumann property and it would Pareto dominate the initial assignment. In consequence, in environments satisfying the Birkhoff-von Neumann property we can directly apply our Full Separation Lemma.

There are multi-unit demand settings in which the Birkhoff-von Neumann property is true such as, for instance, the setting in which each agent buys up to some quantity cap of each object, and two lotteries over bundles are treated as equivalent when they are equivalent as lotteries over the quantities of objects; the equivalence which is natural if each agent $i$ 's utility from a feasible bundle of objects is given by the sum of agent's von NeumannMorgenstern valuations $\tilde{v}_{i}=\left(\tilde{v}_{i}^{1}, \ldots, \tilde{v}_{i}^{|X|}\right)$ for objects in the bundle, that is the utility from bundle $q_{i}=\left(q_{i}^{1}, \ldots, q_{i}^{|X|}\right) \in X_{i}$ is the scalar product $q_{i} \tilde{v}$; the utility from other bundles is zero (cf. Budish et al. 2013). ${ }^{22}$

At the same time, the Birkhoff-von Neumann Theorem does not in general extend to

\footnotetext{
${ }^{21}$ For a taxonomy of bundle prices and discussion of the assumption that the price of the bundle is the sum of item prices, see Bikchandani and Ostroy (2002). They refer to such prices as linear. We also maintain the standard assumption that all agents face the same prices, thus the prices we study are anonymous in their terminology.

${ }^{22}$ Budish et al. (2013) discuss how any profile of random assignments $\left(q_{i}\right)_{i \in I}$ that satisfies the above constraints can be implemented as lotteries over deterministic assignments. They also prove the First Welfare Theorem for the case of equal budgets and additive utilities and showed how to use Milgrom's (2009) integer assignment messages to reduce certain non-linear preferences to this linear setting. The single-unit demand setting is the special case of the multi-unit demand setting, in which $|i|=1$ for each agent $i$. As implied by our discussion of Birkhoff-von Neumann's property, our Second Welfare Theorem remains true for any type of consumption constraints $X_{i}$ that satisfy Birkhoff-von Neumann's property, e.g. because they satisfy Budish et al.'s hierarchy condition or Pycia and Unver's (2015) decomposition conditions.
} 
multi-unit assignments, as pointed out by Budish et al. (2013) in an example with three agents and three objects with unit supply. When each agent demands two objects, then the following random assignment is feasible in expectation but it is not feasible: each agent receiving a pair of objects (different pair for each agent) with probability $\frac{1}{2}$ and receiving no objects with the remaining probability $\frac{1}{2} \cdot{ }^{23}$ The following example illustrates the same failure of the Birkhoff-von Neumann property; the example also illustrates our formalism and lays the ground for the proof of Proposition 1 as well as for Examples 3, 4, and 5.

Example 2. There are four objects with unit supply, and there are two agents, each demanding two objects. Thus, the supply vector is $S=(1,1,1,1)$ and the set of admissible bundles is

$$
B_{1}=B_{2}=\{(1,1,0,0),(1,0,1,0),(1,0,0,1),(0,1,1,0),(0,1,0,1),(0,0,1,1)\}
$$

The random assignment $Q=\left(q_{1}, q_{2}\right)$ where $q_{1}=(1 / 2,0,0,0,0,1 / 2)$ and $q_{2}=(0,0,1 / 2,1 / 2,0,0)$ is feasible in expectation because $\sum_{i \in\{1,2\}, b \in B} b q_{i}^{b}=S$. However, $Q$ is not feasible. We can see this readily as in $Q$ agent 1 receives either both "left objects" 1 and 2 or both "right objects" 3 and 4 , while agent 2 receives either both "extremal" objects 1 and 4 or both "middle" objects 2 and 3. More formally, if there is $\left(\lambda_{D}\right)_{D \in \mathcal{D}} \geq 0, \sum_{D \in \mathcal{D}} \lambda_{D}=1$ meeting the condition in the definition, then there must be $D \in \mathcal{D}$ such that $b_{1}(D)=(1,1,0,0)$ and $\lambda_{D}>0$. However, $\lambda_{D}>0$ implies that $b_{2}(D) \in\{(1,0,0,1),(0,1,1,0)\}$. In either case $D$ generates excess demand for either object 1 or object 2 , contradicting $D \in \mathcal{D}$.

In environments in which the Birkhoff-von Neumann property may fail, formulating the analogue of our Theorem 1 requires care. This is demonstrated by the following

Proposition 1. Not every efficient feasible random assignment $Q^{*}$ is an equilibrium assignment.

Proof. Consider again the two agents and four objects from Example 2, with the set of feasible bundles studied in this example. Assume that $v_{1}=(1,1-\varepsilon, 0,0,1-\varepsilon, 1)$ and $v_{2}=(0,1-\varepsilon, 1,1,1-\varepsilon, 0)$ where $\varepsilon \in\left(0, \frac{1}{2}\right)$. Consider assignment $\left(q_{1}^{*}, q_{2}^{*}\right)$ such that $q_{1}^{*}=$ $(0,1 / 2,0,0,1 / 2,0)$ and $q_{2}^{*}=(0,1 / 2,0,0,1 / 2,0)$ where the probabilities of bundles in $B$ are listed in the same order as the bundles in Example 2. This assignment is feasible because we can implement it as a $\frac{1}{2}: \frac{1}{2}$ lottery between two feasible deterministic assignments: $((1,0,1,0),(0,1,0,1))$ and $((0,1,0,1),(1,0,1,0))$.

\footnotetext{
${ }^{23}$ The failure of the Birkhoff-von Neumann property was further analyzed by Nguyen, Peivandi and Vohra (2016).
} 
The assignment $\left(q_{1}^{*}, q_{2}^{*}\right)$ is also efficient. By way of contradiction, suppose that some other assignment $\left(q_{1}, q_{2}\right)$ Pareto dominates $\left(q_{1}^{*}, q_{2}^{*}\right)$. As the expected utility from the assignment $Q^{*}$ is $1-\varepsilon$ for both agents, we have

$$
\begin{aligned}
& q_{1}^{1}+q_{1}^{6}+(1-\epsilon)\left(q_{1}^{2}+q_{1}^{5}\right) \geq 1-\varepsilon, \\
& q_{2}^{3}+q_{2}^{4}+(1-\epsilon)\left(q_{1}^{2}+q_{1}^{5}\right) \geq 1-\varepsilon
\end{aligned}
$$

where superscripts on probabilities $q_{i}^{1}, \ldots, q_{i}^{6}$ denote the position in which the bundles are listed in $B$. Denoting $\rho_{1} \equiv q_{1}^{1}+q_{1}^{6}=q_{2}^{1}+q_{2}^{6}, \rho_{2} \equiv q_{1}^{3}+q_{1}^{4}=q_{2}^{3}+q_{2}^{4}$, and $\rho_{3} \equiv q_{1}^{2}+q_{1}^{5}=q_{2}^{2}+q_{2}^{5}$, and recognizing that $1-\rho_{3}=\rho_{1}+\rho_{2}$, we can rewrite the above inequalities as

$$
\begin{aligned}
& \rho_{1} \geq\left(1-\rho_{3}\right)(1-\epsilon)=\left(\rho_{1}+\rho_{2}\right)(1-\epsilon), \\
& \rho_{2} \geq\left(1-\rho_{3}\right)(1-\epsilon)=\left(\rho_{1}+\rho_{2}\right)(1-\epsilon) .
\end{aligned}
$$

Because $\varepsilon<1 / 2$, this system of inequalities cannot be satisfied unless $\rho_{1}=\rho_{2}=0$. Hence, $\left(q_{1}, q_{2}\right)$ must put all the weight on the second and fifth bundle, just like $\left(q_{1}^{*}, q_{2}^{*}\right)$, and we can conclude that no feasible random assignment Pareto-dominating $\left(q_{1}^{*}, q_{2}^{*}\right)$.

In spite of being feasible and efficient, $\left(q_{1}^{*}, q_{2}^{*}\right)$ cannot be an equilibrium assignment. Indeed, for any vector of prices $p \in \mathbb{R}_{+}^{|X|}$ the cost of each of the bundles $q_{1}^{*}, q_{2}^{*}, q_{1}=$ $(1 / 2,0,0,0,0,1 / 2)$, and $q_{2}=(0,0,1 / 2,1 / 2,0,0)$ is $\frac{1}{2} \sum_{x} p^{x}$, while $q_{i} \cdot v_{i}>q_{i}^{*} \cdot v_{i}$ for both $i \in\{1,2\}$.

\subsection{Second Welfare Theorem}

In order to recover the Second Welfare Theorem we will strengthen the Pareto efficiency requirement. We say that a feasible random assignment of bundles $Q^{*}$ is strongly efficient if it is not ex-ante Pareto-dominated by any feasible-in-expectation random assignment of bundles. Because every feasible assignment is feasible in expectation, strong efficiency is indeed more demanding than efficiency we studied so far. A positive feature of strong efficiency, and an advantage over the efficiency concept studied above, is that verifying it does not require the market participants to verify whether swaps of probabilities can be implemented; it is the natural concept when thinking in terms of marginal probabilities. In all settings that satisfy the Birkhoff-von Neumann Theorem, strong efficiency and efficiency are of course equivalent.

The following result then holds ${ }^{24}$

\footnotetext{
${ }^{24}$ Combining this result and the previous proposition, we can conclude that in the setting of Example 2 efficiency does not imply strong efficiency.
} 
Theorem 2. (Second Welfare Theorem for General Multi-unit Demands) If a feasible random assignment of bundles $Q^{*}$ is strongly efficient, then it is an equilibrium random assignment supported by some vector of prices $p^{*} \in \mathbb{R}_{+}^{|X|}$ and some vector of budgets $w^{*}=\left(w_{i}^{*}\right)_{i \in I} \in \mathbb{R}_{+}^{|I|}$.

We prove this theorem as an immediate corollary from the following

Theorem 3. If a feasible-in-expectation random assignment of bundles $Q^{*}$ cannot be ex-ante Pareto-dominated by any other feasible-in-expectation random assignment of bundles, then $Q^{*}$ is an equilibrium random assignment supported by some prices $p^{*} \in \mathbb{R}_{+}^{|X|}$ and budgets $\left(w_{i}^{*}\right)_{i \in I} \in \mathbb{R}_{+}^{|I|}$.

The latter result is more general because it only requires random assignment of bundles $Q^{*}$ to be feasible in expectation.

Remark 1. In both of Theorems 2 and 3, we can add that the equilibrium we construct satisfies the following complementary slackness condition: $p^{x *}>0$ implies that there is no excess supply of object $x, \sum_{i \in I} \beta_{i}^{x} q_{i}^{*}=|x|$. To see this suppose that there is an excess supply of object $x$ at assignment $Q^{*}$. If $0<\sum_{i \in I} \beta_{i}^{x} q_{i}^{*}<|x|$ then the set of feasible assignments contains assignments with more of object $x$ than $Q^{*}$ as well as assignments with less of object $x$ than $Q^{*}$. In particular, the separating hyperplane between feasible assignments and dominant assignments contains a line parallel to $x$-axis. Hence, the resulting price vector is orthogonal to $x$-axis and the price of good $x$ is zero. In the remaining case, $0=\sum_{i \in I} \beta_{i}^{x} q_{i}^{*}$, hence $q_{i}^{*}=0$ for all agents $i$, and the efficiency of assignment $Q^{*}$ allows us to set the price of good $x$ at zero without affecting the equilibrium demands of agents.

To get a sense of the proof of Theorem 3, notice that each random assignment over bundles determines the expected assignment of agent $i$ over the underlying goods, $\mu_{i}=\beta_{i} q_{i}$. Because the prices are defined on the underlying goods, every lottery over bundles that leads to the same expected assignment over the underlying goods has the same price. We can also input utility to the expected assignment by recognizing that in the equilibrium an agent buys the lottery over bundles in $B_{i}$ that maximizes the agent's utility among all lotteries of the same price. For every expected assignment $\mu_{i}$ in the convex hull of $B_{i}$ - the convex hull denoted by $C o\left(B_{i}\right)$-we thus define agent $i$ 's utility $V_{i}$ from $\mu_{i}$ as

$$
V_{i}\left(\mu_{i}\right)=\max _{\left\{q \in \Delta\left(B_{i}\right) \mid \beta_{i} q=\mu_{i}\right\}} q \cdot v_{i} .
$$

The following property of this utility function allows us to apply the methods we developed for the single-demand case and prove the second welfare theorem. 


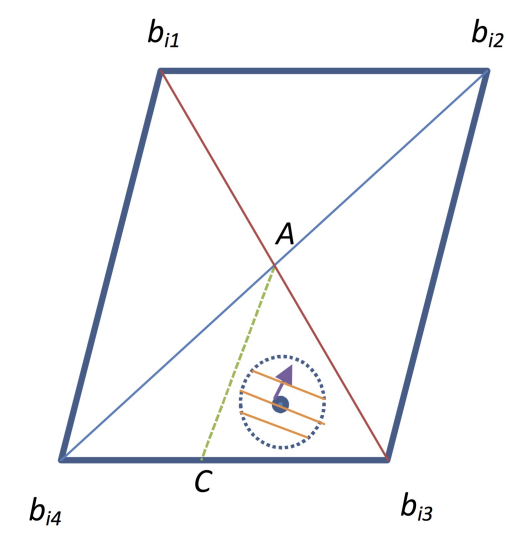

Figure 2: Piece-wise linearity of preferences over expected allocations.

Lemma 2. (Polytope Lemma) For every $\mu_{i} \in \operatorname{Co}\left(B_{i}\right)$, the upper contour set $U_{i}\left(\mu_{i}\right)=$ $\left\{\mu \in \operatorname{Co}\left(B_{i}\right): V_{i}(\mu) \geq V_{i}\left(\mu_{i}\right)\right\}$ of assignments better than $\mu_{i}$ for agent $i$ is a convex polytope.

The proof of this lemma is in Appendix B. The key claim of the lemma is that the upper contour set is a polytope. To get a sense for why this claim is true consider the example illustrated in Figure 2. In the figure, agent $i$ has four possible bundles, $B_{i}=\left\{b_{i 1}, \ldots, b_{i 4}\right\}$, and the the convex hull $C o\left(B_{i}\right)$ takes the shape of the rhomboid. The highlighted dot represents an expected assignment $\mu_{i}$. This expected assignment is a convex combination of $\left\{b_{i 1}, b_{i 3}, b_{i 4}\right\}$ and it is also a convex combination of $\left\{b_{i 2}, b_{i 3}, b_{i 4}\right\}$. Indeed, by the well-known Carathéodory's theorem, any expected assignment in $C o\left(B_{i}\right)$ is a convex combination of just three extreme points in $B_{i}{ }^{25}$ The weights in each of these two convex combinations are unique, and any other representation of $\mu_{i}$ as a convex combination of $\left\{b_{i 1}, b_{i 2}, b_{i 3}, b_{i 4}\right\}$ can be decomposed as a convex combination of these two 3-point convex combinations. Taking into account that $V_{i}\left(\mu_{i}\right)$ is the maximum of a linear function, to calculate $V_{i}\left(\mu_{i}\right)$ we only need to know the utility $V$ at these two 3-point convex combinations. This analysis remains valid for any expected assignment in the interior of the triangle span by points $A, b_{i 3}$, and $b_{i 4}$. Thus, the aforementioned triangle can be divided into a finite number (here: two) of regions on which the set of bundles implementing $V$ is constant. Linearity of the objective function guarantees that there is a hyperplane separating these two regions. If - as in the figure - the expected assignment $\mu_{i}$ is not on this separating hyperplane, then there is a neighborhood of $\mu_{i}$ on which the maximizer convex combination comes from the same set, say $\left\{b_{i 2}, b_{i 3}, b_{i 4}\right\}$. In the figure, this is true for all points in the interior of the triangle span by points $A, C$, and $b_{i 3}$ (note this is a smaller triangle than the one referred to previously). Thus the preferences are linear in a neighborhood of the expected assignment $\mu_{i}$. The figure represents the neighborhood of $\mu_{i}$ by a ball, and it also illustrates the parallel linear

\footnotetext{
${ }^{25}$ We thank Jordi Massó for directing us to the Carathéodory's theorem.
} 
indifference curves and the direction in which utility increases.

Lemma 2 enables us to leverage the methods we developed in Section 3 to prove Theorem 3. The proof, similarly to the proof of Theorem 1, leverages our general Full Separation Lemma (Lemma 1).

Proof of Theorem 3. Let $Y=\left\{m \in \sum_{i \in I} C o\left(B_{i}\right): m \leq S\right\}$ be the set of feasible aggregate expected allocations. Notice that $Y$ is a polytope to which the expected assignment $\mu_{i}^{*}=\sum_{i \in I} \beta_{i} q_{i}^{*}$ of $Q^{*}=\left\{q_{i}^{*}\right\}_{i \in I}$ belongs. Denote the set of aggregate Pareto-improvements by

$Z=\left\{m \in \sum_{i \in I} \operatorname{Co}\left(B_{i}\right) \mid\left(\exists\left(\mu_{i}\right)_{i \in I}\right)\left(\sum_{i \in I} \mu_{i}=m \&(\forall i \in I) V_{i}\left(\mu_{i}\right) \geq V_{i}\left(\mu_{i}^{*}\right) \&(\exists i) V_{i}\left(\mu_{i}\right)>V_{i}\left(\mu_{i}^{*}\right)\right)\right\}$.

Because $Q^{*}$ is not ex-ante Pareto-dominated by any other feasible-in-expectation random assignment, $Z \cap Y=\varnothing$. Furthermore, the aggregate upper contour set $U=\sum_{i \in I} U_{i}\left(\mu_{i}\right)$ is a closure of $Z$ and, by Lemma $2, U$ is a polytope.

To be able to apply our Full Separation Lemma it remains to verify that for no $z \in Z$ and $y \in Y$, there is $\varepsilon>0$ such that $y-\varepsilon(z-y) \in U$. By way of contradiction suppose there are such $z, y$ and $\varepsilon$. Then, there is some $\mu=\left(\mu_{i}\right)_{i \in I}$ such that $\sum_{i \in I} \mu_{i}=y-\varepsilon(z-y)$ and, for all $i \in I, V_{i}\left(\mu_{i}\right) \geq V_{i}\left(\mu_{i}^{*}\right)$. Because $z \in Z$ there is $\mu^{\prime}=\left(\mu_{i}^{\prime}\right)_{i \in I}$ such that $\sum_{i \in I} \mu_{i}^{\prime}=z$ and, for all $i \in I, V_{i}\left(\mu_{i}^{\prime}\right) \geq V_{i}\left(\mu_{i}^{*}\right)$, with strict inequality for some $i$. Consider the expected assignment $\mu^{\prime \prime}=\frac{1}{1+\varepsilon} \mu+\frac{\varepsilon}{1+\varepsilon} \mu^{\prime}$. By construction, $\sum_{i \in I} \mu_{i}^{\prime \prime}=y \leq S$, and, by convexity of $V_{i}$ established in Lemma 2, for all $i \in I$ we have $V_{i}\left(\mu_{i}^{\prime \prime}\right) \geq \frac{1}{1+\varepsilon} V_{i}\left(\mu_{i}\right)+\frac{\varepsilon}{1+\varepsilon} V_{i}\left(\mu_{i}^{\prime}\right) \geq V_{i}\left(\mu_{i}^{*}\right)$, with strict inequality for some $i$. This contradicts the fact that $Q^{*}$ is strongly efficient.

Thus we can apply the Full Separation Lemma to conclude that there is a hyperplane that fully separates $Y$ and $Z$. The rest of the proof is standard and follows the same step as the analogous part of Theorem 1 above. QED

\subsection{First Welfare Theorem}

An immediate question is whether all equilibrium outcomes are strongly efficient? We address this question by proving the First Welfare Theorem for strong efficiency under two assumptions. We assume that every agent buys a lowest-cost (cheapest) among all optimal affordable lotteries, a standard assumption in the analysis of the pseudomarkets introduced and motivated by Hylland and Zeckhauser (1979). The lowest-cost assumption is, for instance, implied by the generic assumption that each agent has a unique favorite bundle, which immediately implies that each agent buys a cheapest favorite affordable bundle. We also restrict attention to equilibria satisfying the complementary slackness condition: $p^{x *}>0$ 
implies that there is no excess supply of $\operatorname{good} x, \sum_{i \in I} \beta_{i}^{x} q_{i}^{*}=|x|{ }^{26}$

Theorem 4. (First Welfare Theorem) Let $Q^{*}$ be an equilibrium assignment with prices $p^{*} \in \mathbb{R}_{+}^{|X|}$ and budgets $\left(w_{i}^{*}\right)_{i \in I} \in \mathbb{R}_{+}^{|I|}$ such that the complementary slackness condition is satisfied and each agent buys one of her lowest-cost optimal affordable lotteries over bundles. Then, $Q^{*}$ is strongly efficient.

Proof. By way of contradiction, suppose $Q^{*}=\left\{q_{i}^{*}\right\}_{i \in I}$ is not strongly efficient. Then there is an expected allocation $\left(\mu_{i}\right)_{i \in I}$ such that $\sum_{i \in I} \mu_{i} \leq S$ and $V_{i}\left(\mu_{i}\right) \geq q_{i}^{*} \cdot v_{i}$ for all $i \in I$, with at least one inequality strict. If an agent $i$ is not satiated under $q_{i}^{*}$ - that is with positive probability her outcome is worse than her most preferred bundle - then $p^{*} \cdot \mu_{i} \geq p^{*} \cdot \beta_{i} q_{i}^{*}$ by the same argument that works in standard Walrasian equilibrium theory with non-satiated agents. $^{27}$ If agent $i$ is satiated then the same inequality holds provided she bought the least expensive most-preferred lottery. The same argument, gives us $p^{*} \cdot \mu_{i}>p^{*} \cdot \beta_{i} q_{i}^{*}$ for agents $i$ for whom the inequality $V_{i}\left(\mu_{i}\right) \geq q_{i}^{*} \cdot v_{i}$ is strict. Summing up the inequalities over agents, we obtain $\sum_{i \in I} p^{*} \cdot \mu_{i}>\sum_{i \in I} p^{*} \cdot \beta_{i} q_{i}^{*}$. In particular, there is an object $x$ with positive price $p^{x *}>0$ and such that $\sum_{i \in I} \mu_{i}^{x}>\sum_{i \in I} \beta_{i}^{x} q_{i}^{*}$. Because $p^{x *}>0$, the complementary slackness assumption implies that $\sum_{i \in I} \beta_{i}^{x} q_{i}^{*}=|x|$ (no excess supply). We thus obtain a contradiction with the assumption that $\sum_{i \in I} \mu_{i} \leq S$. QED

\subsection{Existence}

The final question we address is whether strongly efficient assignments - and hence equilibriaexist. The potential subtlety is that strongly efficient feasible random assignment need to be favorable compared to both feasible and unfeasible random assignment of bundles. It turns out that in general a feasible random assignment of bundles that is strongly efficient might not exist. However, strongly efficient assignments exist in course allocation setting, the leading example of the multi-unit setting, provided agents' preferences over bundles are

\footnotetext{
${ }^{26}$ The assumption of complementary slackness is justified as we show that all strongly efficient assignments can be implemented via equilibria satisfying complementary slackness, cf. Remark 1 . Complementary slackness is however a more substantive assumption than in environments with local non-satiation where it is trivially satisfied. The reason to impose the lowest cost assumption can be seen in the following example with one unit of object $x$, two units of object $y$ and three agents. Agent 1 strictly prefers $x$ to $y$, agent 2 strictly prefers $y$ to $x$, and agent 3 is indifferent. The following is an equilibrium in which each of these agents has budget of 1 : the price of good $x$ is 2 , the price of good $y$ is 0 , agents 1 and 3 buy probability .5 in good $x$ and probability .5 in good $y$, agent 2 buys probability 1 in good $y$. This equilibrium violates the lowest-cost assumption and it is inefficient as it is dominated by an allocation in which agent 1 buys probability 1 of good $\mathrm{x}$, while each of the remaining agents buy probability 1 of good $\mathrm{y}$.

${ }^{27}$ Suppose $p^{*} \cdot \mu_{i}<p^{*} \cdot \beta_{i} q_{i}^{*}$ and let $b_{i}$ be a most preferred bundle of agent $i$. We can then find a small weight $\alpha>0$ such that $V_{i}\left(\alpha b_{i}+(1-\alpha) \mu_{i}\right)>q_{i}^{*} \cdot v_{i}$ and $p^{*} \cdot\left(\alpha b_{i}^{*}+(1-\alpha) \mu_{i}\right) \leq p^{*} \cdot \beta_{i} q_{i}^{*}$, contradicting that $q_{i}^{*}$ was an optimal choice in $i$ 's budget set.
} 
strict; strict preferences are generic. ${ }^{28}$

Theorem 5. (Existence) In course allocation, if agents' preferences over bundles are strict, then there exists a feasible deterministic assignment that is strongly efficient.

Proof. We construct the strongly efficient assignment via a serial dictatorship mechanism. Fix an arbitrary ordering of agents, say $1, \ldots,|I|$. Let 0 denote the bundle with 0 units of each object. Let $B_{1}^{*} \subseteq B_{1}$ be the set of bundles $b$ such that $(b, 0, \ldots, 0)$ is feasible; we assign to agent 1 his or her most preferred bundle $b_{1}$ in $B_{1}^{*}$. Let $B_{2}^{*} \subseteq B_{2}$ be the set of bundles $b$ such that $\left(b_{1}, b, 0, \ldots, 0\right)$ is feasible; we assign to agent 2 his or her most preferred bundle $b_{2}$ in $B_{2}^{*}$. Proceeding recursively, we define a feasible deterministic assignment. Note that the mechanism is well-defined because in the course allocation setting $B_{1}^{*}, B_{2}^{*}, \ldots, B_{|I|}^{*}$ are non-empty and, thanks to the strict preference assumption, each agent $i$ has the most preferred bundle in $B_{i}^{*}$.

It remains to verify that the resulting assignment is strongly efficient. By way of contradiction suppose that $Q=\left(q_{i}\right)_{i \in I}$ is a strictly dominant random assignment that is feasible in expectation. Agent by agent, we show that $q_{i}$ put probability 1 on assignment $b_{i}$ constructed above. Consider agent 1 . If bundle $\tilde{b} \in B_{1}-B_{1}^{*}$ then the failure of feasibility would imply that $\tilde{b}^{x}>|x|$ for some object $x \in X$; this cannot happen in course allocation where $\tilde{b}^{x} \leq 1$ and $|x| \geq 1$. Thus, the support of the lottery $q_{1}$ is contained in $B_{1}^{*}$. Furthermore, because $b_{1}$ is 1 's the unique most favorite bundle in $B_{1}^{*}$ and the support of $q_{1}$ is contained in $B_{1}^{*}$, Pareto efficiency implies that $q_{1}$ puts entire probability on $b_{1}$.

Consider agent 2. If bundle $\tilde{b} \in B_{2}-B_{2}^{*}$ then $\tilde{b}^{x}>|x|-b_{1}^{x}$ for some object $x \in X$; this can only happen when $b_{1}^{x}=1$ and $|x|-b_{1}^{x}=0$. As $Q$ is feasible in expectation, the expected number of units of $x$ in $q_{2}$ is zero, and we conclude that $\tilde{b}^{x}=0$ for all bundles in the support of $q_{2}$. Thus, the support of $q_{2}$ is contained in $B_{2}^{*}$. Furthermore, because $b_{2}$ is $i_{2}$ 's the unique most favorite bundle in $B_{2}^{*}$ and the support of $q_{2}$ is contained in $B_{2}^{*}$, Pareto efficiency implies that $q_{2}$ puts entire probability on $b_{2}$.

Proceeding recursively, we find that the analysis for agent 3 and for subsequent agents is analogous to the analysis for agent 2 . If bundle $\tilde{b} \in B_{i}-B_{i}^{*}$ then $\tilde{b}^{x}>|x|-b_{1}^{x}-\ldots-b_{i-1}^{x}$ for some object $x \in X$; this can only happen when $b_{i}^{x}=1$ and $|x|-b_{1}^{x}-\ldots-b_{i-1}^{x}=0$. We conclude that the support of $q_{i}$ is contained in $B_{i}^{*}$ and hence that $q_{i}$ puts entire probability on $b_{i}$. In effect, $Q$ puts probability 1 on $\left(b_{1}, \ldots, b_{|I|}\right)$ which is the assignment of the serial dictatorship mechanism. Hence, $Q$ does not strictly Pareto dominate the outcome of the mechanism; a contradiction that proves the assignment of the serial dictatorship is strongly efficient. QED

\footnotetext{
${ }^{28}$ We illustrate the role of the course allocation and strict preference assumptions in Examples 4 and 5 below. The restriction to course allocation was missing in our 2017-2020 drafts.
} 
Remark 2. The proof of Theorem 5 shows not only that there exists strongly efficient assignments but also that the serial dictatorships used in the proof always generate strongly efficient outcomes.

The existence theorem is less straightforward than it might ex ante appear. First, not all Pareto efficient deterministic outcomes are strongly efficient, even in course allocation setting with strict preferences. Second, the strong efficiency of serial dictatorships is not guaranteed in general multi-unit environments. Third, the assumption of strict preferences is needed for the result. These three points are illustrated by the following examples.

Example 3. To see that not all Pareto efficient deterministic outcomes are strongly efficient, consider the environment of Example 2 allowing that bundles in which agents receive fewer than two courses are also admissible. ${ }^{29}$ Suppose that - in the terminology from Example 2 -agent 1 strictly prefers the left bundle $(1,1,0,0)$ to the right bundle $(0,0,1,1)$ and strictly prefers the latter to all other bundles. Suppose that agent 2 strictly prefers the extreme bundle $(1,0,0,1)$ to the middle bundle $(0,1,1,0)$, strictly prefers the middle bundle to the left bundle, and strictly prefers the latter to all other bundles. Then, the deterministic assignment in which agent 1 receives the right bundle and agent 2 receives the left bundle is Pareto efficient. At the same time, this deterministic assignment is not strongly efficient because it is dominated by the feasible-in-expectation random assignment $\left(q_{1}, q_{2}\right)$ from Example 2, which gives the 50-50 lottery over left and right bundle to agent 1 and gives 50-50 lottery over extreme and middle bundle to agent 2 .

Example 4. To see how some feasibility constraints may cause existence problems, let us modify Example 2 so that

$$
B_{1}=\{(1,1,0,0),(1,0,0,1),(0,0,1,1)\}, \quad B_{2}=\{(1,1,0,0),(1,0,0,1),(0,1,1,0)\} .
$$

Then, only two deterministic assignments are feasible: either agent 1 receives $(1,0,0,1)$ while agent 2 receives $(0,1,1,0)$; or agent 1 receives $(0,0,1,1)$ while agent 2 receives $(1,1,0,0)$. If the valuation vectors satisfy $v_{1}^{(1,1,0,0)}>v_{1}^{(0,0,1,1)}>v_{1}^{(1,0,0,1)}$ and $v_{2}^{(1,0,0,1)}>v_{2}^{(0,1,1,0)}>v_{2}^{(1,1,0,0)}$, then, for each agent, the value of any feasible deterministic assignment is strictly lower than the expected value of the random assignment $\left(q_{1}, q_{2}\right)$ from Example 2; the random assignment $\left(q_{1}, q_{2}\right)$ is feasible in expectation but it is not feasible. In effect, in this modification of Example 2, no feasible assignment is strongly efficient.

\footnotetext{
${ }^{29}$ The admissibility of these bundles plays no role in the argument but the course allocation model requires it.
} 
Example 5. To see the need for the strict preference assumption, consider again Example 2 allowing that bundles in which agents receive fewer than two courses are also admissible. Fix $\varepsilon \in\left(0, \frac{1}{2}\right)$, and let the values of admissible bundles be equal to 0 , except for

$$
\begin{aligned}
& v_{1}^{(1,1,0,0)}=v_{1}^{(0,0,1,1)}=v_{2}^{(1,0,0,1)}=v_{2}^{(0,1,1,0)}=1, \\
& v_{1}^{(1,0,1,0)}=v_{1}^{(0,1,0,1)}=v_{2}^{(1,0,1,0)}=v_{2}^{(0,1,0,1)}=1-\varepsilon .
\end{aligned}
$$

As $\varepsilon>0$, all deterministic assignments - and hence all feasible random assignments - are Pareto dominated by the feasible-in-expectation random assignment $\left(q_{1}, q_{2}\right)$ from from Example 2. Indeed, $\left(q_{1}, q_{2}\right)$ gives expected utility 1 to each agent, while any deterministic assignment either gives utility $1-\varepsilon$ to both agents, or it gives utility 0 to both agents, or it gives utility 0 to one of the agents and 1 to the other. In particular, in this example no feasible assignment is strongly efficient. ${ }^{30}$

\subsection{Constraints}

Our model allows for many design constraints such as e.g. reserving some seats in a school for a group of applicants, while allowing all applicants to compete for the remaining seats; to model such constraint we create an auxiliary object "reserved seats" and we define the sets $B_{i}$ in such a way that individual allocations with copies of the reserved seats object are feasible only for the selected group of applicants.

Furthermore, our First and Second Welfare Theorems (Theorems 1-4) remain valid — with no changes in proofs - under any conjunction of linear constraints imposed on random and deterministic assignments as long as the set of feasible assignments remains nonempty. ${ }^{31}$ Our existence result (Theorem 5) and its proof remain valid under any conjunction of nonnegative integer upper bounds on unweighted sums of probabilities of arbitrarily selected agents receiving arbitrarily selected objects.

\footnotetext{
${ }^{30}$ This example hinges on indifferences and, unlike Example 3, it is non-generic; indeed, Theorem 5 implies that generically at least one deterministic assignment is strongly efficient.

${ }^{31}$ Indeed, under any such conjunction of constraints the polytopes in the proofs of our Second Welfare Theorems (Theorems 1, 2, and 3) remain polytopes and all the steps of the proofs and all our lemmas, including our Full Separation Lemma, remain applicable. The proof of the First Welfare Theorem (Theorem 4) remains valid because imposing a conjunction of linear constraints preserves the convexity of sets we work with; for the role of convexity in this proof see footnote 27.
} 


\section{Conclusion}

We have established the Second Welfare Theorem for the general class of single-unit demand and multi-unit demand discrete allocation problems. We show that in large range of market design settings - including school choice, course allocation, and refugee resettlementefficient assignments can be implemented by token price mechanisms, thus providing the foundations for the literature's focus on such mechanisms. Our Second Welfare Theorem has already played the role of a revelation principle for discrete mechanism design. ${ }^{32}$

In addition to this substantive insight, we developed a novel approach to analyzing Walrasian markets in which agents' preferences fail the standard local non-satiation assumption; our approach builds on the polytope properties of the Walrasian markets for discrete resources.

Our analysis allows arbitrary utility profiles over bundles of objects and arbitrary linear constraints, thus contributing both to the literature on constraints in market design as well as the literature on complementarities and substitutes discussed in the introduction.

\section{A Proof of Lemma 1 (Full Separation Lemma)}

We say that $\bar{Z}$ is partially separated (or simply, separated) from $Y$ when there is scalar $w \in \mathbb{R}$ and price vector $p \in \mathbb{R}^{n}$ such that $p \cdot \bar{z} \geq w \geq p \cdot y$ for all $\bar{z} \in \bar{Z}$ and $y \in Y$. We say that $Z$ is fully separated from $Y$ when there is scalar $w \in \mathbb{R}$ and price vector $p \in \mathbb{R}^{n}$ such that $p \cdot z>w \geq p \cdot y$ for all $z \in Z$ and $y \in Y$.

Let $P$ be a polytope in $\mathbb{R}^{n}$ that is the intersection of a finite number of half spaces; each half-space bounded by a hyperplane. Let $H_{1}, \ldots, H_{K}$ be the set of these hyperplanes; we refer to them as the hyperplanes defining $P$. A face of $P$ is an intersection $P \cap\left(\cap_{k \in J} H_{k}\right)$ for some $J \subseteq\{1, \ldots, K\}$, and we also call the empty set a face of $P .{ }^{33}$ The affine hull of a set, denoted aff, is the collection of all finite linear combinations of points in the set with weights adding up to 1 (with negative weights allowed, as opposed to a convex hull). In the proof we will use McLennan's (2002) Separating Hyperplane Theorem, which states the following: ${ }^{34}$

Lemma 3. (McLennan's Separating Hyperplane Theorem) Suppose $Y \subset \mathbb{R}^{n}$ and $\bar{Z} \subset \mathbb{R}^{n}$ are polyhedra. Let $F_{Y}$ be the intersection of all faces of $Y$ that contain $Y \cap \bar{Z}$ and let $F_{\bar{Z}}$ be the intersection of all faces of $\bar{Z}$ that contain $Y \cap \bar{Z}$. If aff $\left(F_{Y} \cup F_{\bar{Z}}\right) \neq \mathbb{R}^{n}$, then

\footnotetext{
${ }^{32}$ Cf. Miralles and Pycia (2015), Miralles (2017) and Schlegel and Mamageishvili (2019), as well as other papers discussed in the introduction.

${ }^{33}$ Notice that we allow $J=\emptyset$ and hence $P$ is a face of itself.

${ }^{34}$ McLennan developed this theorem in an ordinal context unrelated to the problems studied in our paper, and it was never previously used to analyze Walrasian equilibria.
} 
there is a hyperplane $H$ that separates $\mathbb{R}^{n}$ into two half spaces $H^{+}$and $H^{-}$where $Y \subseteq H^{-}$ and $\bar{Z} \subseteq H^{+}$such that $Y \cap H=F_{Y}$ and $\bar{Z} \cap H=F_{\bar{Z}}$.

As a consequence we conclude

Lemma 4. Suppose $Y \subset \mathbb{R}^{n}$ and $\bar{Z} \subset \mathbb{R}^{n}$ are polyhedra. Let $F_{Y}$ be the intersection of all faces of $Y$ that contain $Y \cap \bar{Z}$ and let $F_{\bar{Z}}$ be the intersection of all faces of $\bar{Z}$ that contain $Y \cap \bar{Z}$. Either there exists a hyperplane $H$ that separates $\mathbb{R}^{n}$ into two half spaces $H^{+}$and $H^{-}$ where $Y \subseteq H^{-}$and $\bar{Z} \subseteq H^{+}$such that $Y \cap H=F_{Y}$ and $\bar{Z} \cap H=F_{\bar{Z}}$, or else aff $\left(F_{Y} \cup F_{\bar{Z}}\right)=\mathbb{R}^{n}$ and $Y=F_{Y}$ and $\bar{Z}=F_{\bar{Z}}$.

Proof of Lemma 4. If $\operatorname{aff}\left(F_{Y} \cup F_{\bar{Z}}\right) \neq \mathbb{R}^{n}$ then the claim follows from McLennan's Lemma. It remains to consider the case when $\operatorname{aff}\left(F_{Y} \cup F_{\bar{Z}}\right)=\mathbb{R}^{n}$. Suppose we embed $Y$ and $\bar{Z}$ in $\mathbb{R}^{n} \times \mathbb{R}$ as $Y \times\{0\}$ and $\bar{Z} \times\{0\}$, respectively. Then, McLennan's Lemma implies the existence of a hyperplane $H$ that separates $\mathbb{R}^{n+1}$ into two half spaces $H^{+}$and $H^{-}$where $Y \times\{0\} \subseteq H^{-}$ and $\bar{Z} \times\{0\} \subseteq H^{+}$such that $Y \times\{0\} \cap H=F_{Y} \times\{0\}$ and $\bar{Z} \times\{0\} \cap H=F_{\bar{Z}} \times\{0\}$. The two inclusions allow us to infer that $Y \times\{0\} \subseteq H$ and $\bar{Z} \times\{0\} \subseteq H$. Furthermore, the two equalities and aff $\left(F_{Y} \cup F_{\bar{Z}}\right)=\mathbb{R}^{n}$ allows us to conclude that $H=\mathbb{R}^{n} \times\{0\}$. The claim of Lemma 5 then follows. QED

We now turn to the proof of our Full Separation Lemma. We may assume that $Y \cap \bar{Z}$ is non-empty as otherwise the lemma follows from the standard separating hyperplane theorem for closed convex sets. ${ }^{35}$ Let $S$ be the affine hull of $Y \cap \bar{Z}$. Being an affine hull, $S$ is a linear subspace of $\mathbb{R}^{n}$. Furthermore, $S$ is a linear subspace of dimension lower than $n$. Indeed, if not then the convexity of $Y \cap \bar{Z}$ would imply that there is an open ball $B \subset Y \cap \bar{Z}$ around some point $y^{*} \in Y \cap \bar{Z}$. But then, taking any $z \in Z$ and setting $\delta=z-y^{*}$, we would find an $\epsilon>0$ such that $y^{*}-\epsilon \delta \in B$ contrary to $y^{*}-\epsilon \delta \notin \bar{Z}$.

Let $F_{\bar{Z}}$ be the intersection of all faces of $\bar{Z}$ that contain $Y \cap \bar{Z}$, that is $F_{\bar{Z}}$ is the intersection of $\bar{Z}$ with all hyperplanes that define faces of $\bar{Z}$ and contain $\bar{Z} \cap Y$. Similarly, let $F_{Y}$ be the intersection of all faces of $Y$ that contain $Y \cap \bar{Z}$. From Lemma 4, we know that either (i) there exists a hyperplane $H$ that separates $\mathbb{R}^{n}$ into two half spaces $H^{+}$and $H^{-}$where $Y \subseteq H^{-}$and $\bar{Z} \subseteq H^{+}$such that $Y \cap H=F_{Y}$ and $\bar{Z} \cap H=F_{\bar{Z}}$, or else (ii) aff $\left(F_{Y} \cup F_{\bar{Z}}\right)=\mathbb{R}^{n}$ and $Y=F_{Y}$ and $\bar{Z}=F_{\bar{Z}}$.

Consider case (i). Because $Z \cap H \subseteq Z \cap F_{\bar{Z}}$, to prove that $H$ fully separates $Z$ and $Y$, it is sufficient to show that $Z \cap F_{\bar{Z}}=\varnothing$. Suppose not. $F_{\bar{Z}}$ is non-empty. If $F_{\bar{Z}}$ is a singleton then let $z$ be the only point contained in $F_{\bar{Z}}$. Because $\bar{Z} \cap Y=F_{\bar{Z}} \cap Y$ is nonempty, we conclude that $z \in Y$ and because $Z \cap F_{\bar{Z}}$ is non-empty we conclude that $z \in Z$. But this

\footnotetext{
${ }^{35}$ The theorem says that there is a fully separating hyperplane for any two disjoint convex closed sets in $\mathbb{R}^{n}$, see e.g. Boyd and Vandenberghe (2004).
} 
contradicts $Z \cap Y=\varnothing$. We can thus assume that $F_{\bar{Z}}$ contains at least two points. Define the relative interior of a set to be the interior of this set in the linear space spanned by the affine hull of this set. Because $F_{\bar{Z}}$ is a convex polytope, its relative interior, denoted $\operatorname{ri}\left(F_{\bar{Z}}\right)$, is nonempty. Because $F_{\bar{Z}}$ is the intersection of $\bar{Z}$ and all the hyperplanes $H_{k}$ defining $\bar{Z}$ and containing $Y \cap \bar{Z}$, we can infer that $Y \cap \operatorname{ri}\left(F_{\bar{Z}}\right) \neq \varnothing$. Indeed, if $Y \cap \operatorname{ri}\left(F_{\bar{Z}}\right)=\varnothing$ then the intersection $Y \cap \bar{Z}=Y \cap F_{\bar{Z}}$ of the polytopes $Y$ and $\bar{Z}$ would be disjoint with the relative interior of $F_{\bar{Z}}$ and hence, being convex, this intersection would be contained in a face of $F_{\bar{Z}}$ that is a proper subset of $F_{\bar{Z}}$. But this is a contradiction as $F_{\bar{Z}}$ is the smallest face of $\bar{Z}$ containing $Y \cap \bar{Z}$. Let thus $a \in Y \cap \operatorname{ri}\left(F_{\bar{Z}}\right)$, and, by way of contradiction, assume that there is $z^{*} \in Z \cap F_{\bar{Z}}$. Because $z^{*} \in F_{\bar{Z}}$ and $a \in \operatorname{ri}\left(F_{\bar{Z}}\right)$, we infer that $a-\varepsilon\left[z^{*}-a\right] \in F_{\bar{Z}} \subseteq \bar{Z}$ for any $\varepsilon>0$ small enough, and the assumptions of our lemma imply that $a+\left[z^{*}-a\right] \notin Z$, a contradiction.

Finally, we show that case (ii) cannot happen. If it did then $\bar{Z}=F_{\bar{Z}}$ and hence $\bar{Z}$ itself would be the only face of $\bar{Z}$ that contains $Y \cap \bar{Z}$. Because $Y$ is convex, this would imply that $Y$ has a non-empty intersection with the relative interior of $\bar{Z}$. Let $a \in Y \cap \operatorname{ri}(\bar{Z})$ and let $z^{*} \in Z$. Because $z^{*} \in \bar{Z}$ and $a \in \operatorname{ri}(\bar{Z})$, we infer that $a-\varepsilon\left[z^{*}-a\right] \in \bar{Z}$ for any $\varepsilon>0$ small enough, and the assumptions of our lemma imply that $a+\left[z^{*}-a\right] \notin Z$, a contradiction that concludes the proof of the Full Separation Lemma. QED

\section{B Proof of Lemma 2 (Polytope Lemma)}

The next two lemmas jointly imply the result.

Lemma 5. (Convexity) Preferences represented by $V_{i}$ are convex.

Proof. Take $\lambda \in[0,1]$ and $\mu_{i}, \mu_{i}^{\prime} \in \operatorname{Co}\left(B_{i}\right)$. We need to show that $\lambda V_{i}\left(\mu_{i}\right)+(1-$ $\lambda) V_{i}\left(\mu_{i}^{\prime}\right) \leq V_{i}\left(\lambda \mu_{i}+(1-\lambda) \mu_{i}^{\prime}\right)$. By the definition of $V$, there is $q \in \Delta\left(B_{i}\right)$ such that $\beta_{i} q=\mu_{i}$ and $V_{i}\left(\mu_{i}\right)=q \cdot v_{i}$. Similarly, there is $q^{\prime} \in \Delta\left(B_{i}\right)$ such that $\beta_{i} q^{\prime}=\mu_{i}^{\prime}$ and $V_{i}\left(\mu_{i}^{\prime}\right)=q^{\prime} \cdot v_{i}$. Then,

$$
\begin{aligned}
& \lambda V_{i}\left(\mu_{i}\right)+(1-\lambda) V_{i}\left(\mu_{i}^{\prime}\right) \\
= & {\left[\lambda q+(1-\lambda) q^{\prime}\right] \cdot v_{i} } \\
\leq & \max _{\left\{q^{\prime \prime} \in \Delta\left(B_{i}\right) \mid \beta_{i} q^{\prime \prime}=\lambda \mu_{i}+(1-\lambda) \mu_{i}^{\prime}\right\}} q^{\prime \prime} \cdot v_{i} \\
= & V_{i}\left(\lambda \mu_{i}+(1-\lambda) \mu_{i}^{\prime}\right)
\end{aligned}
$$

where the inequality follows because $\beta_{i}\left[\lambda q+(1-\lambda) q^{\prime}\right]=\lambda \mu_{i}+(1-\lambda) \mu_{i}^{\prime}$, and hence $q^{\prime \prime}=$ $\lambda q+(1-\lambda) q^{\prime}$ is in the set the maximum above is taken over. QED 
Lemma 6. (Local Affinity) Let $i$ be an agent. Let $L$ be the linear space spanned by $B_{i}$ and let $d$ be its dimension. For almost every $\mu_{i} \in C o\left(B_{i}\right)$, there exists a convex L-neighborhood $M \subseteq C o\left(B_{i}\right)$ of $\mu_{i}$ such that $V_{i}$ is an affine function of $\mu$ on $M$; that is, for all $\mu, \mu^{\prime} \in M$ and $\lambda \in[0,1], V_{i}\left(\lambda \mu+\left(1-\lambda \mu^{\prime}\right)\right)=\lambda V_{i}(\mu)+(1-\lambda) V_{i}\left(\mu^{\prime}\right)$.

Proof. The set $D$ of expected assignments in $C o\left(B_{i}\right)$ that can be represented as a convex combination of $d$ or fewer points in $B_{i}$ is of measure zero in $L$. This claim follows from two observations. First, the convex hull of any $d$ or fewer points is of dimension at most $d-1$, and hence of measure zero in the $d$-dimensional space $L$. Second, there is only a finite number of subsets in $B_{i}$ because $B_{i}$ itself is finite.

Let us fix an expected assignment $\mu_{i} \in C o\left(B_{i}\right)-D$. Let $\mathcal{B}_{i}\left(\mu_{i}\right)$ be the set of all $B \subseteq B_{i}$ such that $|B| \leq d+1$ and $\mu_{i}$ is a convex combination of elements from $B$. Because $\mu_{i} \notin D$ we infer that each $B \in \mathcal{B}_{i}\left(\mu_{i}\right)$ has exactly $d+1$ elements. $\mathcal{B}_{i}\left(\mu_{i}\right)$ is finite because $B_{i}$ is finite. $\mathcal{B}_{i}\left(\mu_{i}\right)$ is nonempty because Carathéodory's Theorem tells us that $\mu_{i}$ can be represented as a convex combination of $d+1$ elements of $B_{i}$. Furthermore, for any $B \in \mathcal{B}_{i}\left(\mu_{i}\right)$ there is exactly one convex combination of elements of $B$ that gives $\mu_{i}$. Indeed, if there were two such convex combinations then $\mu_{i}$ would also be a convex combination of elements from a proper subset of $B$; a contradiction because $|B|=d+1$ and $\mu_{i} \notin D$.

By definition of $V_{i}$, there is $B \in \mathcal{B}_{i}\left(\mu_{i}\right)$ such that $V_{i}\left(\mu_{i}\right)=q \cdot v_{i}$ for some $q \in \Delta\left(B_{i}\right)$ such that $\mu_{i}=\beta_{i} q_{i}$, and $q^{b}>0$ iff $b \in B$. Let us denote by $\mu^{1}, \ldots, \mu^{d+1}$ the expected assignments that belong to $B$. For any $\varepsilon \in\left(0, \min \left\{q^{b} \mid b \in B\right\} \cup\left\{1-q^{b} \mid b \in B\right\}\right)$, the set $B^{\varepsilon}$ of convex combinations of elements of $B$ with weight on each $b \in B$ taken from $\left(q^{b}-\varepsilon, q^{b}+\varepsilon\right)$ is a convex full-dimensional open subset of $C o\left(B_{i}\right)$, and hence a convex $L$-neighborhood of $\mu_{i}$.

We claim that for sufficiently small $\varepsilon>0$, all expected assignments in $B^{\varepsilon}$ have a unique decomposition as a convex combination over a subset of $\mathcal{B}_{i}\left(\mu_{i}\right)$, and this unique decomposition is over $B$. Indeed, if not then there is a sequence of $\mu_{i}^{\ell} \in C o\left(B_{i}\right)$ that tends to $\mu_{i}$ as $\ell \rightarrow \infty$ and such that all $\mu_{i}^{\ell}$ have at least two convex decompositions over subsets of $\mathcal{B}_{i}\left(\mu_{i}\right)$. Same argument as above shows that then all $\mu_{i}^{\ell} \in D$ and we can select a subsequence $\ell_{n}$ such that all $\mu_{i}^{\ell_{n}}$ are convex combinations of the same $d$ (or fewer) points in $B$. But then $\mu_{i}=\lim _{n \rightarrow \infty} \mu_{i}^{\ell_{n}}$ would also be a convex combination of the same $d$ (or fewer) points in $B$, a contradiction.

Take $\varepsilon$ that is sufficiently small in the sense of the above claim. Then, $\mu_{i}$ is an arbitrary element of the full measure subset of $C o\left(B_{i}\right)$, and the uniqueness of the convex decomposition implies that for all $q \in \Delta\left(B_{i}\right)$ such that $q^{b}>0$ iff $b \in B$ and $\beta_{i} q$ belongs to the convex neighborhood $M=B^{\varepsilon}$ of $\mu_{i}$, the utility $V_{i}\left(\beta_{i} q\right)=q \cdot v_{i}$. Thus, $V_{i}$ is affine on $M$. QED 


\section{References}

[1] Arrow, Kenneth (1951). "An Extension of the Basic Theorems of Classical Welfare Economics." In: Proceedings of the Second Berkeley Symposium on Mathematical Statistics and Probability. University of California Press.

[2] Abdulkadiroglu, Atila, Nikhil Agarwal, and Parag A. Pathak (2017). "The Welfare Effects of Coordinated Assignment: Evidence from the New York City High School Match." American Economic Review 107(12): 3635-3689.

[3] Abdulkadiroglu, Atila, Che Yeon-Koo, and Yasuke Yasuda (2011). "Resolving Conflicting Preferences in School Choice: the Boston Mechanism Reconsidered," American Economic Review 101(1), 399-410.

[4] Abdulkadiroglu, Atila, Parag A. Pathak, and Alvin E. Roth (2009). "Strategy-proofness versus Efficiency in Matching with Indifferences: Redesigning the NYC High School Match." American Economic Review 99(5), 1954-1978.

[5] Abdulkadirglu, Atila and Tayfun Sonmez (1998). "Random Serial Dictatorship and the Core from Random Endowments in House Allocation Problems." Econometrica 66, 689-701.

[6] Abdulkadirglu, Atila and Tayfun Sonmez (2003). "School Choice: A Mechanism Design Approach." American Economic Review 93(3), 729-747.

[7] Anderson, Robert M. (1988). "The Second Welfare Theorem with Nonconvex Preferences." Econometrica 56(2), 361-382.

[8] Andersson, Tommy, and Lars Ehlers (2020). "Assigning Refugees to Landlords in Sweden: Efficient Stable Maximum Matchings." Scandinavian Journal of Economics 122(3), 937-965.

[9] Ashlagi, Itai, and Peng Shi (2016). "Optimal Allocation Without Money: an Engineering Approach." Management Science 62(4), 1078-1097.

[10] Azevedo E. and E. Budish (2019). "Strategyproofness in the Large." The Review of Economic Studies 86(1), 81-116.

[11] Babaioff, Moshe, Noam Nisan, and Inbal Talgam-Cohen (2018). "Competitive Equilibrium with Indivisible Goods and Generic Budgets." Working Paper.

[12] Baldwin, Elizabeth, and Paul Klemperer (2019). "Understanding Preferences: "Demand Types", and the Existence of Equilibrium With Indivisibilities." Econometrica 87(3), 867932.

[13] Baldwin, Elizabeth, Omer Edhan, Ravi Jagadeesan, Paul Klemperer, and Alexander Teytelboym (2020). "The Equilibrium Existence Duality: Equilibrium with Indivisibilities and Income Effects." Working Paper. 
[14] Bergstrom, Ted C. (1976). "How to Discard 'Free Disposability' - at No Cost," Journal of Mathematical Economics, 3, 131-134.

[15] Bikhchandani, Sushil, and Joseph M. Ostroy (2002). "The Package Assignment Model." Journal of Economic Theory 107 (2), 377-406.

[16] Bogomolnaia, A. and H. Moulin (2001). "A New Solution to the Random Assignment Problem." Journal of Economic Theory 100, 295-328.

[17] Bogomolnaia, A., H. Moulin, F. Sandomirskiy, and E. Yanovskaya (2017). "Competitive division of a mixed manna." Econometrica 85(6), 1847-1871.

[18] Bogomolnaia, A., H. Moulin, F. Sandomirskiy, and E. Yanovskaya (2019). "Dividing bads under additive utilities." Social Choice and Welfare 52(3), 395-417.

[19] Boyd S. and L. Vandenberghe (2004). Convex Optimization. Cambridge University Press.

[20] Budish E. (2011). "The Combinatorial Assignment Problem: Approximate Competitive Equilibrium from Equal Incomes," Journal of Political Economy 119, 1061-1103.

[21] Budish E. and E. Cantillon (2012). "The Multi-unit Assignment Problem: Theory and Evidence from Course Allocation at Harvard," American Economic Review 102, 2237-2271.

[22] Budish. E., Y.K. Che, F. Kojima, and P. Milgrom (2013). "Designing Random Allocation Mechanisms: Theory and Applications," American Economic Review 103, 585-623.

[23] Carroll, Gabriel (2010). "An Efficiency Theorem for Incompletely Known Preferences." Journal of Economic Theory 145, 2463-2470.

[24] Debreu, Gerard (1951). "The Coefficient of Resource Utilization." Econometrica, 19(3), 273292.

[25] Delacretaz, David, Scott Duke Kominers, and Alexander Teytelboym (2020). "Matching Mechanisms for Refugee Resettlement." Working Paper.

[26] Echenique Federico, Antonio Miralles, and Jun Zhang (2019a). "Fairness and Efficiency for Probabilistic Allocations with Endowments," Working Paper.

[27] Echenique, Federico, Antonio Miralles, and Jun Zhang (2019b). "Constrained Pseudo-market Equilibrium". Working Paper.

[28] Featherstone, Clayton and Muriel Niederle (2016). "Improving on Strategy-proof School Choice Mechanisms: An Experimental Investigation." Games and Economic Behavior 100, 353-375.

[29] Florig M. and J. Rivera (2010). "Core equivalence and welfare properties without divisible goods." Journal of Mathematical Economics 46, 467-474. 
[30] Gul F., W. Pesendorfer, and M. Zhang (2019). "Market Design and Walrasian Equilibrium." Working Paper.

[31] Hafalir I. and A. Miralles (2015). "Welfare Maximizing Assignment of Agents to Hierarchical Positions." Journal of Mathematical Economics 61, 253-270.

[32] Hara, Chiaki (2005). "Existence of Equilibria in Economies with Bads." Econometrica 73(2), 647-658.

[33] He Y., A. Miralles, M. Pycia, and J. Yan. (2018). "A Pseudo-Market Approach to Assignment with Priorities." American Economic Journal: Microeconomics 10(3), 272-314.

[34] Hylland, A. and R. Zeckhauser (1979). "The Efficient Allocation of Individuals to Positions," Journal of Political Economy 87, 293-314.

[35] Kamada, Yuichiro, and Fuhito Kojima (2015). "Efficient Matching Under Distributional Constraints: Theory and Applications." American Economic Review 105, 67-99.

[36] Makowski, L., J. M. Ostroy, and U. Segal (1999) "Efficient Incentive Compatible Economies Are Perfectly Competitive," Journal of Economic Theory, 85, 169-225.

[37] Manelli, Alejandro M. (1991). "Monotonic Preferences and Core Equivalence." Econometrica $59(1), 123-138$

[38] Manjunath, Vikram (2014). "Markets for Fractional Partnerships," Working Paper.

[39] Mas-Colell, Andreu (1992). "Equilibrium Theory with Possibly Satiated Preferences." In: Equilibrium and Dynamics, Essays in Honor of David Gale, edited by Mukul Majumdar. New York: St. Martins' Press, 201-213.

[40] Mas-Colell A., M. D. Whinston, and J. Green (1995). Microeconomic Theory. Oxford University Press.

[41] Maskin, Eric S., and Kevin W. S. Roberts (2008). "On the Fundamental Theorems of General Equilibrium." Economic Theory 35, 233-240.

[42] McLennan, Andrew (2002). "Ordinal Efficiency and the Polyhedral Separating Hyperplane Theorem." Journal of Economic Theory 105, 435-449.

[43] McLennan, Andrew (2018). "Efficient Disposal Equilibria of Pseudomarkets." Working Paper.

[44] Milgrom, Paul R. (2009). "Assignment messages and exchanges." American Economic Journal: Microeconomics, 1(2), 95-113.

[45] Miralles, Antonio (2008). "School Choice: The Case for the Boston Mechanism." Working Paper. 
[46] Miralles, Antonio (2017). "Ex-ante Efficiency in Assignments with Seniority Rights." Review of Economic Design 21, 33-48.

[47] Miralles, Antonio, and Marek Pycia (2015). "Large vs. Continuum Assignment Economies." Working Paper.

[48] Miralles, Antonio, and Marek Pycia (2017). "Prices and Efficient Assignments Without Transfers. Online Appendix." Working Paper.

[49] Nguyen T., Peivandi A. and Vohra R. (2016). "Assignment problems with complementarities." Journal of Economic Theory 165, 209-241.

[50] Ostrovsky, Michael (2008). "Stability in Supply Chain Networks." American Economic Review 98(3), 897-923.

[51] Pareto, Vilfredo (1909). Manuel d'Economie Politique, Paris: M. Giard.

[52] Pycia Marek (2012). "Stability and Preference Alignment in Matching and Coalition Formation." Econometrica 80(1), 323-362.

[53] Pycia, Marek (2014). "The Cost of Ordinality." Working Paper.

[54] Pycia, Marek 2019. "Evaluating with Statistics: Which Outcome Measures Differentiate Among Matching Mechanisms?" Working Paper.

[55] Pycia, M., and U. Unver (2015). "Decomposing Random Mechanisms." Journal of Mathematical Economics 61, 21-33.

[56] Reny, Philip J. (2017). "Assignment Problems." Journal of Political Economy 125(6), 19031914.

[57] Richter, Michael, and Ariel Rubinstein (2015). "Back to Fundamentals: Equilibrium in Abstract Economies," American Economic Review 105(8), 2570-2594.

[58] Schlegel, Jan Christoph, and Akaki Mamageishvili (2019). "Welfare Theorems for Random Assignments with Priorities." Working Paper.

[59] Sun, Ning, and Zaifu Yang (2006). "Equilibria and indivisibilities: gross substitutes and complements." Econometrica 74, 1385-1402.

[60] Sonmez, Tayfun, and Utku Unver (2010). "Course Bidding at Business Schools." International Economic Review 51(1), 99-123.

[61] Vazirani, Vijay V., and Mihalis Yannakakis (2020). "Computational Complexity of the Hylland-Zeckhauser Scheme for One-Sided Matching Markets. Working Paper. 